\title{
Successive Convex Approximation Algorithms for Sparse Signal Estimation with Nonconvex Regularizations
}

\author{
Yang Yang, Marius Pesavento, Symeon Chatzinotas and Björn Ottersten
}

\begin{abstract}
In this paper, we propose a successive convex approximation framework for sparse optimization where the nonsmooth regularization function in the objective function is nonconvex and it can be written as the difference of two convex functions. The proposed framework is based on a nontrivial combination of the majorization-minimization framework and the successive convex approximation framework proposed in literature for a convex regularization function. The proposed framework has several attractive features, namely, i) flexibility, as different choices of the approximate function lead to different types of algorithms; ii) fast convergence, as the problem structure can be better exploited by a proper choice of the approximate function and the stepsize is calculated by the line search; iii) low complexity, as the approximate function is convex and the line search scheme is carried out over a differentiable function; iv) guaranteed convergence to a stationary point. We demonstrate these features by two example applications in subspace learning, namely, the network anomaly detection problem and the sparse subspace clustering problem. Customizing the proposed framework by adopting the bestresponse type approximation, we obtain soft-thresholding with exact line search algorithms for which all elements of the unknown parameter are updated in parallel according to closed-form expressions. The attractive features of the proposed algorithms are illustrated numerically.
\end{abstract}

Index Terms-Big Data, Line Search, Majorization Minimization, Nonconvex Regularization, Successive Convex Approximation

\section{INTRODUCTION}

In this paper, we consider the following optimization problem

$$
\underset{\mathbf{x} \in \mathcal{X} \subseteq \mathbb{R}^{K}}{\operatorname{minimize}} h(\mathbf{x}) \triangleq f(\mathbf{x})+g(\mathbf{x}),
$$

where $f$ is a smooth function and $g$ is a nonsmooth function, and the constraint set $\mathcal{X}$ is closed and convex. Such a formulation plays a fundamental role in parameter estimation, and typically $f$ models the estimate error while $g$ is a regularization (penalty) function promoting in the solution a certain structure known a priori such as sparsity [1]. Among others, the linear regression problem is arguably one of the most extensively studied problems and it is a special case of (2) by setting $f(\mathbf{x})=\frac{1}{2}\|\mathbf{A} \mathbf{x}-\mathbf{y}\|_{2}^{2}$ and $g(\mathbf{x})=\lambda\|\mathbf{x}\|_{1}$, where $\mathbf{A} \in \mathbb{R}^{N \times K}$ is a known dictionary and $\mathbf{y} \in \mathbb{R}^{K \times 1}$ is the available noisy measurement. Many algorithms have been proposed for the linear regression problem, for example, the fast iterative softthresholding algorithm (FISTA) [2], the block coordinate descent (BCD) algorithm [3], the alternating direction method of multiplier (ADMM) [4], proximal algorithm [5] and the parallel BCD algorithm with exact line search [6].

In linear regression, the function $f(\mathbf{x})=\frac{1}{2}\|\mathbf{A x}-\mathbf{y}\|_{2}^{2}$ is convex in $\mathbf{x}$. This is generally desirable in the design of numerical algorithms solving problem (1) iteratively. However, this desirable property is not available in many other applications where we have to deal with a nonconvex $f$. Consider for example the linear regression model where

Y. Yang, S. Chatzinotas and B. Ottersten are with Interdisciplinary Centre for Security, Reliability and Trust, University of Luxembourg, L1855 Luxembourg (email: yang.yang@uni.lu, symeon.chatzinotas@uni.lu, bjorn.ottersten@uni.lu). Their work is supported by the ERC project AGNOSTIC.

M. Pesavento is with Communication Systems Group, Technische Universität Darmstadt, 64283 Darmstadt, Germany (email: pesavento@nt.tudarmstadt.de). His work is supported by the EXPRESS Project within the DFG Priority Program CoSIP (DFG-SPP 1798). we assume that the dictionary $\mathbf{A}$ is unknown and treated as a variable. In this case, the objective function $f(\mathbf{A}, \mathbf{x})=\frac{1}{2}\|\mathbf{A} \mathbf{x}-\mathbf{y}\|_{2}^{2}$ is a nonconvex function in $(\mathbf{A}, \mathbf{x})$ and the problem is known as Dictionary Learning. An additional difficulty is that the gradient of $f(\mathbf{A}, \mathbf{x})$ with respect to (w.r.t.) $(\mathbf{A}, \mathbf{x})$ is no longer Lipschitz continuous. In nonlinear regression problems [7], $f(\mathbf{x})$ is in general a nonconvex function, for example, $f(\mathbf{x})=\frac{1}{2}\|\boldsymbol{\sigma}(\mathbf{A x})-\mathbf{b}\|_{2}^{2}$ and $\boldsymbol{\sigma}$ is a given function specifying the nonlinear regression model, e.g., the cosine or sigmoid function.

When the function $f$ is nonconvex, the above mentioned algorithms must be re-examined. For example, the FISTA algorithm no longer converges, and the generalized iterative soft-thresholding algorithm (GIST) has been proposed instead [7]. However, as a proximal type algorithm, the GIST algorithm suffers from slow convergence [2]. The block coordinate descent (BCD) algorithm usually exhibits a faster convergence because the variable update is based on the socalled nonlinear best-response [8]: the variable $\mathbf{x}$ is partitioned into multiple block variables $\mathbf{x}=\left(\mathbf{x}_{k}\right)_{k=1}^{K}$, and in each iteration of the BCD algorithm, one block variable, say $\mathbf{x}_{k}$, is updated by its bestresponse $\mathbf{x}_{k}^{t+1}=\arg \min _{\mathbf{x}_{k}} h\left(\mathbf{x}_{1}^{t+1}, \ldots, \mathbf{x}_{k-1}^{t+1}, \mathbf{x}_{k}, \mathbf{x}_{k+1}^{t}, \ldots, \mathbf{x}_{K}^{t}\right)$ (i.e., the optimal point that minimizes $h(\mathbf{x})$ w.r.t. the variable $\mathbf{x}_{k}$ only while the remaining variables are fixed to their values of the preceding iteration) while all block variables are updated sequentially. Its convergence is guaranteed under some sufficient conditions on $f$ and $g[3,9,10,11]$, and due to its simplicity, this method and its variants have been successfully adopted to many practical problems including the network anomaly detection problem in [12]. Nevertheless, a major drawback of the sequential update is that it may incur a large delay because the $(k+1)$-th block variable $\mathbf{x}_{k+1}$ cannot be updated until the $k$-th block variable $\mathbf{x}_{k}$ is updated and the delay may be very large when $K$ is large, which is a norm rather than an exception in big data analytics [13].

A parallel variable update based on the best-response (also known as the Jacobi algorithm [8] or the parallel BCD algorithm [14]) seems attractive as a mean to speed up the updating procedure, however, sufficient conditions guaranteeing the convergence of a parallel BCD algorithm are known for smooth problems only (that is, $g(\mathbf{x})=0$ ) and they are rather restrictive, for example, $f$ is convex and satisfies the diagonal dominance condition [8]. However, it has been shown in some recent works $[9,14,15,16]$ that if a stepsize is employed in the variable update, the convergence conditions can be notably relaxed, for example, $f$ could be nonconvex. There the notion of approximate functions play a fundamental role: a sequence of successively refined approximate problems are solved, and the algorithm converges to a stationary point of the original problem (1) for a number of choices of approximate functions, including the best-response type approximation, as long as they satisfy some assumptions on, e.g., (strong or strict) convexity, hence the name of the successive convex approximation (SCA) framework $[9,15,16]$.

The performance of the SCA algorithms in $[15,16]$ is largely dependent on the choice of the stepsizes, namely, exact/successive line search and diminishing stepsizes such as constant stepsizes and diminishing stepsizes. The exact line search scheme (also known as minimization rule) and the successive line search scheme (also 
known as the Armijo rule) are widely used for smooth problems (see [17]) and adopted in [9]. However, when the traditional exact line search is directly extended to the nonsmooth problems, a nonconvex nonsmooth optimization problem must be solved and the complexity is thus high (see for example [14, Sec. III-D]). The successive line search has a lower complexity, but it typically consists of evaluating the nonsmooth function $g$ several times for different stepsizes per iteration [16, Remark 4], which might be computationally expensive for some $g$ such as the nuclear norm [18] because of the large dimension. Diminishing stepsizes has the lowest complexity, but sometimes they are difficult to deploy in practice because the convergence behavior is sensitive to the decay rate [6]. As a matter of fact, the applicability of SCA algorithms in big data analytics is severely limited by the meticulous choice of stepsizes [13]. Furthermore, to apply the diminishing stepsizes, the function $f$ must have a Lipschitz continuous gradient (i.e., there exists a finite constant $L_{\nabla f}$ such that $\|\nabla f(\mathbf{x})-\nabla f(\mathbf{y})\| \leq L_{\nabla f}\|\mathbf{x}-\mathbf{y}\|$ for all $\left.\mathbf{x}, \mathbf{y} \in \mathcal{X}\right)$, and this assumption is not satisfied for many applications, for example in Dictionary Learning where $f(\mathbf{A}, \mathbf{x})=\frac{1}{2}\|\mathbf{A} \mathbf{x}-\mathbf{y}\|_{2}^{2}$ and $\mathcal{X}=\mathbb{R}^{K}$.

To reduce the complexity of the traditional line search schemes and avoid the parameter tuning of the diminishing stepsize rules, an efficient line search scheme is proposed in [6]: the exact line search is carried out over a properly constructed differentiable function, while in the successive line search, the approximate function only needs to be optimized once. The line search schemes in [6] are much easier to implement, and closed-form expressions exist for many applications. Besides this, the assumption on the strong or strict convexity of the approximate functions made in $[15,16]$ is also relaxed to convexity in [6].

Another popular algorithm for problem (1) in big data analytics is the alternating direction method of multipliers (ADMM) [4], but it does not have a guaranteed convergence to a stationary point if the optimization problem (1) is nonconvex [19]. There is some recent development in ADMM for nonconvex problems, see [20,21] and the references therein. Nevertheless, the algorithms proposed therein are for specific problems and not applicable in a broader setup. For example, the ADMM algorithm proposed in [20] is designed for nonconvex sharing/consensus problems, and the ADMM algorithm proposed in [21] converges only when the dictionary matrix has full row rank, which is generally not satisfied for the network anomaly detection problem [12].

So far we have assumed that the regularization function $g$ in (1) is convex, for example, the $\ell_{1}$-norm function, as it has been used as a standard regularization function to promote sparse solutions [22]. However, it was pointed out in $[23,24]$ that the $\ell_{1}$-norm is a loose approximation of the $\ell_{0}$-norm and it tends to produce biased estimates when the sparse signal has large coefficients. A more desirable regularization function is singular at the origin while flat elsewhere. Along this direction, several nonconvex regularization functions have been proposed, for example, the smoothly clipped absolute deviation [23], the capped $\ell_{1}$-norm [25], and the logarithm function [26]; we refer the interested reader to [27] for a more comprehensive review.

The nonconvexity of the regularization function $g$ renders many of the above discussed algorithms inapplicable, including the SCA framework [6], because the nonsmooth function $g$ is assumed to be convex. It is shown in [27] that if the smooth function $f$ is convex and the nonconvex regularization function $g$ can be written as the sum of a convex and a concave function, the classic majorization-minimization (MM) method can be applied to find a stationary point of (1): firstly in the majorization step, an upper bound function is obtained by linearizing the concave regularization function, and then the upper bound function is minimized in the minimization step; see [28] for a recent overview article on the MM algorithms. Nevertheless, the minimum of the upper bound cannot be expressed by a closed-form expression and must be found iteratively. The MM method is thus a two-layer algorithm that involves iterating within iterations and has a high complexity: a new instance of the upper bound function is minimized by iterative algorithms at each iteration of the MM method while minimizing the upper bound functions repeatedly is not a trivial task, even with a warm start that sets the optimal point of the previous instance as the initial point of the new instance.

To reduce the complexity of the classic MM method, an upper bound function based on the proximal type approximation is designed in [29] and it is much easier to optimize (see [30] for a more general setup). Although the algorithm converges to a stationary point, it suffers from several limitations. Firstly, the convergence speed with the proximal type upper bound functions is usually slower than some other approximations, for example, the best-response approximation [6]. Secondly, the proximal type upper bound function minimized in each iteration is nonconvex, and it may not be easy to optimize except in the few cases discussed in [29]. Thirdly, the function $f$ must have a Lipschitz continuous gradient.

In this paper, we study problem (1) and focus on the case that the smooth function $f$ is nonconvex and the nonsmooth nonconvex regularization function $g$ is the difference of two convex functions; ${ }^{1}$ a recent work [33] shows that such a decomposition exists for a large class of nonconvex regularization functions $g$. We propose a SCA framework and it is based on a nontrivial combination of the SCA framework for a convex $g$ proposed in [6] and standard MM framework [28]. In particular, in each iteration, we first construct a (possibly nonconvex) upper bound of the original function $h$ by the standard MM method, and then minimize a convex approximation of the upper bound which can be constructed by the standard SCA framework [6]. On the one hand, this is a beneficial combination because the approximate function is typically much easier to minimize than the original upper bound function and the proposed algorithm is thus a single layer algorithm if we choose an approximate function such that its minimum has a closed-form expression. On the other hand, this is a challenging combination because the convergence of the proposed algorithms can no longer be proved by existing techniques. To further speed up the convergence, we design a line search scheme to calculate the stepsize with an affordable complexity by generalizing the line search schemes proposed in [6] for a convex $g$. The proposed framework has several attractive features, namely,

- flexibility, as the approximate function does not have to be a global upper bound of the original objective function and different choices of the approximate functions lead to different types of algorithms, for example, proximal type approximation and best-response type approximation;

- fast convergence, as the problem structure can be better exploited by a proper choice of the approximate function, and the stepsize is calculated by the line search;

- low complexity, as the approximate function is convex and easy to optimize, and the proposed line search scheme over a properly constructed differentiable function is easier to implement than traditional schemes which are directly applied to the original nonconvex nonsmooth objective function;

- guaranteed convergence to a stationary point, even when the function $f$ does not have a Lipschitz continuous gradient, as long as the approximate function is convex and satisfies some other mild assumptions on gradient consistency and continuity.

We then illustrate the above attractive features by customizing the proposed framework for two example applications in subspace learning, namely, the network anomaly detection problem and the sparse

${ }^{1}$ Some preliminary results of this paper have been presented at $[31,32]$. 
subspace clustering problem, where both the optimal point of the (best-response type) approximate functions and the stepsize obtained from the exact line search have closed-form expressions.

The rest of the paper is organized as follows. In Sec. II we introduce the problem formulation and the example applications. The novel SCA framework is proposed and its convergence is analyzed in Sec. III. In Sec. IV and Sec. V, two example applications, the network anomaly detection problem through sparsity regularized rank minimization and the subspace clustering problem through capped $\ell_{1}$ norm minimization, are discussed, both theoretically and numerically. The paper is concluded in Sec. VI.

Notation: We use $x, \mathbf{x}$ and $\mathbf{X}$ to denote a scalar, vector and matrix, respectively. We use $X_{j k}$ to denote the $(j, k)$-th element of $\mathbf{X} ; x_{k}$ is the $k$-th element of $\mathbf{x}$ where $\mathbf{x}=\left(x_{k}\right)_{k=1}^{K}$, and $\mathbf{x}_{-k}$ denotes all elements of $\mathbf{x}$ except $x_{k}: \mathbf{x}_{-k}=\left(x_{j}\right)_{j=1, j \neq k}^{K}$. We denote $\mathbf{x}^{-1}$ as the element-wise inverse of $\mathbf{x}$, i.e., $\left(\mathbf{x}^{-1}\right)_{k}=1 / x_{k}$. Notation $\mathbf{x} \circ \mathbf{y}$ and $\mathbf{X} \otimes \mathbf{Y}$ denotes the Hadamard product between $\mathbf{x}$ and $\mathbf{y}$, and the Kronecker product between $\mathbf{X}$ and $\mathbf{Y}$, respectively. The operator $[\mathbf{x}]_{\mathbf{a}}^{\mathbf{b}}$ returns the element-wise projection of $\mathbf{x}$ onto $[\mathbf{a}, \mathbf{b}]:[\mathbf{x}]_{\mathbf{a}}^{\mathbf{b}} \triangleq$ $\max (\min (\mathbf{x}, \mathbf{b}), \mathbf{a})$. We denote $\mathbf{d}(\mathbf{X})$ as the vector that consists of the diagonal elements of $\mathbf{X}$. We denote $\operatorname{diag}(\mathbf{x})$ and $\operatorname{diag}(\mathbf{X})$ as a diagonal matrix whose diagonal elements are as same as those of $\mathbf{x}$ and $\mathbf{X}$, respectively. We use $\mathbf{1}$ to denote a vector with all elements equal to 1 . The sign function $\operatorname{sign}(x)=1$ if $x>0,0$ if $x=0$, and -1 if $x<0$, and $\operatorname{sign}(\mathbf{x})=\left(\operatorname{sign}\left(x_{k}\right)\right)_{k}$.

\section{Problem Formulation}

In this section, we formally introduce the problem that will be tackled in the rest of the paper. In particular, we assume $g(\mathbf{x})$ in (1) can be written as the difference of two convex functions, and consider from now on the following problem:

$$
\underset{\mathbf{x} \in \mathcal{X}}{\operatorname{minimize}} h(\mathbf{x}) \triangleq f(\mathbf{x})+\underbrace{g^{+}(\mathbf{x})-g^{-}(\mathbf{x})}_{g(\mathbf{x})},
$$

where

- $f$ is a proper and differentiable function with a continuous gradient,

- $g^{+}$and $g^{-}$are convex functions, and

- $\mathcal{X}$ is a closed and convex set.

Note that $f(\mathbf{x})$ is not necessarily convex, and $g^{+}(\mathbf{x})$ and $g^{-}(\mathbf{x})$ are not necessarily differentiable.

We aim at developing efficient iterative algorithms that converge to a stationary point $\mathbf{x}^{\star}$ of problem (2) that satisfies the first order optimality condition:

$$
\left(\mathbf{x}-\mathbf{x}^{\star}\right)^{T}\left(\nabla f\left(\mathbf{x}^{\star}\right)+\boldsymbol{\xi}^{+}\left(\mathbf{x}^{\star}\right)-\boldsymbol{\xi}^{-}\left(\mathbf{x}^{\star}\right)\right) \geq 0, \forall \mathbf{x} \in \mathcal{X},
$$

where $\boldsymbol{\xi}^{+}(\mathbf{x})$ and $\boldsymbol{\xi}^{-}(\mathbf{x})$ is a subgradient of $g^{+}(\mathbf{x})$ and $g^{-}(\mathbf{x})$, respectively. Note that a convex function always has a subgradient.

\section{A. Example Application: Network Anomaly Detection Through Spar- sity Regularized Rank Minimization}

Consider the problem of estimating a low rank matrix $\mathbf{X} \in \mathbb{R}^{N \times K}$ and a sparse matrix $\mathbf{S} \in \mathbb{R}^{I \times K}$ from the noisy measurement $\mathbf{Y} \in$ $\mathbb{R}^{N \times K}$ which is the output of a linear system:

$$
\mathbf{Y}=\mathbf{X}+\mathbf{D S}+\mathbf{V}
$$

where $\mathbf{D} \in \mathbb{R}^{N \times I}$ is known and $\mathbf{V}^{N \times K}$ is the unknown noise.

The rank of $\mathbf{X}$ is much smaller than $N$ and $K$, i.e, $\operatorname{rank}(\mathbf{X}) \ll$ $\min (N, K)$, and the support size of $\mathbf{S}$ is much smaller than $I K$, i.e., $\|\mathbf{S}\|_{0} \ll I K$. A natural measure for the estimation error is the least square loss function augmented by regularization functions to promote the rank sparsity of $\mathbf{X}$ and support sparsity of $\mathbf{S}$ :

$$
\underset{\mathbf{X}, \mathbf{S}}{\operatorname{minimize}} \frac{1}{2}\|\mathbf{X}+\mathbf{D S}-\mathbf{Y}\|_{F}^{2}+\lambda\|\mathbf{X}\|_{*}+\mu\|\mathbf{S}\|_{1},
$$

where $\|\mathbf{X}\|_{*}$ is the nuclear norm of $\mathbf{X}$. Problem (3) plays a fundamental role in the analysis of traffic anomalies in large-scale backbone networks [12]. In this application, $\mathbf{D}$ is a given binary routing matrix, $\mathbf{X}=\mathbf{R Z}$ where $\mathbf{Z}$ is the unknown traffic flows over the time horizon of interest, and $\mathbf{S}$ is the traffic volume anomalies. The matrix $\mathbf{X}$ inherits the rank sparsity from $\mathbf{Z}$ because common temporal patterns among the traffic flows in addition to their periodic behavior render most rows/columns of $\mathbf{Z}$ linearly dependent and thus low rank, and $\mathbf{S}$ is assumed to be sparse because traffic anomalies are expected to happen sporadically and last shortly relative to the measurement interval, which is represented by the number of columns $K$.

Problem (3) is convex and it can be solved by the SCA algorithm proposed in [18], which is a parallel BCD algorithm with exact line search. Although it presents a much lower complexity than standard methods such as proximal type algorithms and BCD algorithms, it may eventually become inefficient due to the use of complex models: computing the nuclear norm $\|\mathbf{X}\|_{*}$ has a cubic complexity and is unaffordable when the problem dimension is large. Furthermore, problem (3) is not suitable for the design of distributed and/or parallel algorithms because the nuclear norm $\|\mathbf{X}\|_{*}$ is neither differentiable nor decomposable among the blocks of $\mathbf{X}$ (unless $\mathbf{X}$ is Hermitian).

It follows from the identity $[34,35]$

$$
\|\mathbf{X}\|_{*}=\min _{(\mathbf{P}, \mathbf{Q})} \frac{1}{2}\left(\|\mathbf{P}\|_{F}^{2}+\|\mathbf{Q}\|_{F}^{2}\right) \text {, s.t. } \mathbf{P Q}=\mathbf{X}
$$

that the low rank matrix $\mathbf{X}$ can be written according to the above matrix factorization as the product of two low rank matrices $\mathbf{P} \in$ $\mathbb{R}^{N \times \rho}$ and $\mathbf{Q} \in \mathbb{R}^{\rho \times K}$ for a $\rho$ that is larger than the rank of $\mathbf{X}$ but usually much smaller than $N$ and $K: \operatorname{rank}(\mathbf{X}) \leq \rho \ll \min (N, K)$. It may be useful to consider the following optimization problem where the nuclear norm $\|\mathbf{X}\|_{*}$ is replaced by $\|\mathbf{P}\|_{F}^{2}+\|\mathbf{Q}\|_{F}^{2}$, which is differentiable and separable among its blocks:

$$
\underset{\mathbf{P}, \mathbf{Q}, \mathbf{S}}{\operatorname{minimize}} \frac{1}{2}\|\mathbf{P Q}+\mathbf{D S}-\mathbf{Y}\|_{F}^{2}+\frac{\lambda}{2}\left(\|\mathbf{P}\|_{F}^{2}+\|\mathbf{Q}\|_{F}^{2}\right)+\mu\|\mathbf{S}\|_{1} .
$$

This optimization problem is a special case of (2) obtained by setting

$$
\begin{aligned}
f(\mathbf{P}, \mathbf{Q}, \mathbf{S}) & \triangleq \frac{1}{2}\|\mathbf{P Q}+\mathbf{D S}-\mathbf{Y}\|_{F}^{2}+\frac{\lambda}{2}\left(\|\mathbf{P}\|_{F}^{2}+\|\mathbf{Q}\|_{F}^{2}\right), \\
g^{+}(\mathbf{S}) & \triangleq \mu\|\mathbf{S}\|_{1}, \text { and } g^{-}(\mathbf{S})=0 .
\end{aligned}
$$

Although problem (4) is nonconvex, every stationary point of (4) is an optimal solution of (3) under some mild conditions [19, Prop. 1]. We remark that the gradient of $f(\mathbf{P}, \mathbf{Q}, \mathbf{S})$ w.r.t. $(\mathbf{P}, \mathbf{Q}, \mathbf{S})$ is not Lipschitz continuous.

Another way to enhance (3) is to replace the convex nuclear norm of $\mathbf{X}$ by a general nonconvex regularization function:

$$
\lambda \sum_{n=1}^{N} \kappa\left(\sigma_{n}(\mathbf{X})\right)
$$

where $\kappa(x)$ is a concave and increasing function w.r.t. $x \geq 0$ with $\kappa(0)=0$ while $\nabla \kappa$ is Lipschitz continuous and the number of points at which $\nabla \kappa$ is not differentiable is finite. Note that in (5) we have assumed w.l.o.g. that $N<K$. If $\kappa(x)=x$, (5) reduces to the nuclear norm $\lambda\|\mathbf{X}\|_{*}=\lambda \sum_{n=1}^{N} \sigma_{n}(\mathbf{X})$ in (3). In general, $\kappa\left(\sigma_{n}(\mathbf{X})\right)$ is a nonconvex function of $\mathbf{X}$ and some examples of $\kappa$ are given in [33, Table I]. 
Given the nonconvex regularization function (5), problem (3) is modified in the following problem:

$$
\underset{\mathbf{X}, \mathbf{S}}{\operatorname{minimize}} \frac{1}{2}\|\mathbf{X}+\mathbf{D S}-\mathbf{Y}\|_{F}^{2}+\lambda \sum_{n=1}^{N} \kappa\left(\sigma_{n}(\mathbf{X})\right)+\mu\|\mathbf{S}\|_{1} .
$$

It is a special case of (2) obtained by setting

$$
\begin{aligned}
f(\mathbf{X}, \mathbf{S}) & =\frac{1}{2}\|\mathbf{X}+\mathbf{D S}-\mathbf{Y}\|_{F}^{2} \\
g^{+}(\mathbf{X}, \mathbf{S}) & =\lambda \kappa_{0}\|\mathbf{X}\|_{*}+\mu\|\mathbf{S}\|_{1}, \\
g^{-}(\mathbf{X}) & =\lambda \kappa_{0}\|\mathbf{X}\|_{*}-\lambda \sum_{n=1}^{N} \kappa\left(\sigma_{n}(\mathbf{X})\right),
\end{aligned}
$$

where $\kappa_{0} \triangleq \nabla \kappa(0)$ and $g^{-}(\mathbf{X}, \mathbf{S})$ is convex and differentiable [33, Prop. 5].

In Sec. IV, we will customize the proposed SCA framework to design an iterative soft-thresholding with exact line search algorithm for problems (4) and (6), which is essentially a parallel BCD algorithm.

\section{B. Example Application: Sparse Subspace Clustering Through Capped $\ell_{1}$-Norm Minimization}

Consider the linear regression model

$$
\mathbf{y}=\mathbf{A} \mathbf{x}+\mathbf{v},
$$

where the dictionary $\mathbf{A} \in \mathbb{R}^{N \times K}$ is known and $\mathbf{y} \in \mathbb{R}^{N \times 1}$ is the noisy measurement. To estimate $\mathbf{x}$ which is known to be sparse a priori, we minimize the quadratic estimation error function augmented by some regularization function to promote the sparsity of $\mathrm{x}$. A common routine is to use the $\ell_{1}$-norm, which has however been shown to yield biased estimates for large coefficients [25]. Alternatives include for example the capped $\ell_{1}$-norm function [25, 27, 29], and the resulting optimization problem is as follows:

$$
\underset{\mathbf{x}}{\operatorname{minimize}} \frac{1}{2}\|\mathbf{A x}-\mathbf{y}\|_{2}^{2}+\mu \sum_{k=1}^{K} \min \left(\left|x_{k}\right|, \theta\right) .
$$

This optimization problem is a special case of (2) obtained by setting

$$
\begin{aligned}
f(\mathbf{x}) & \triangleq \frac{1}{2}\|\mathbf{A} \mathbf{x}-\mathbf{b}\|_{2}^{2}, \\
g^{+}(\mathbf{x}) & \triangleq \mu \sum_{k=1}^{K}\left|x_{k}\right|, \text { and } g^{-}(\mathbf{x}) \triangleq \mu \sum_{k=1}^{K}\left|x_{k}\right|-\min \left(\left|x_{k}\right|, \theta\right),
\end{aligned}
$$

where $g^{-}(\mathbf{x})$ is a convex but nonsmooth function. A graphical illustration of the functions $g, g^{+}$and $g^{-}$is provided in Fig. 1, and interested readers are referred to [27, Fig. 2] for more examples.

When $\theta$ is sufficiently large, problem (7) reduces to the standard LASSO problem, which plays a fundamental role in sparse subspace clustering problems [36] and can be solved efficiently by the SCA algorithm proposed in [6]. In Problem (7), we take one step further by considering the capped $\ell_{1}$-norm and then in Sec. V, we customize the proposed SCA framework to design an iterative soft-thresholding with exact line search algorithm for problem (7), which is essentially a parallel BCD algorithm.

\section{The Proposed Successive Convex Approximation ALGORITHMS}

In this section, we formally introduce the proposed SCA framework for problem (2), where $h$ is in general a nonconvex function since $f$ is not necessarily convex and $g^{-}$is convex, and $h$ is in

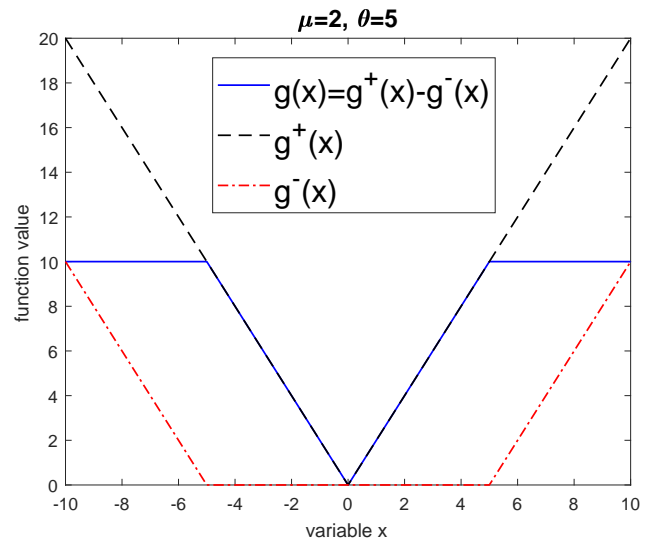

Figure 1. Illustration of the capped $\ell_{1}$-norm function and its decomposition

general a nonsmooth function since both $g^{+}$and $g^{-}$are assumed to be nonsmooth.

At any arbitrary but given point $\mathbf{x}^{t}$, assume the subgradient of $g^{-}(\mathbf{x})$ is $\boldsymbol{\xi}^{-}\left(\mathbf{x}^{t}\right)$. Since $g^{-}$is convex, it follows from the definition of convex functions that

$$
g^{-}(\mathbf{x}) \geq g^{-}\left(\mathbf{x}^{t}\right)+\left(\mathbf{x}-\mathbf{x}^{t}\right)^{T} \boldsymbol{\xi}^{-}\left(\mathbf{x}^{t}\right), \forall \mathbf{x} \in \mathcal{X}
$$

Define $\bar{h}\left(\mathbf{x} ; \mathbf{x}^{t}\right)$ as

$$
\bar{h}\left(\mathbf{x} ; \mathbf{x}^{t}\right) \triangleq f(\mathbf{x})-g^{-}\left(\mathbf{x}^{t}\right)-\left(\mathbf{x}-\mathbf{x}^{t}\right)^{T} \boldsymbol{\xi}^{-}\left(\mathbf{x}^{t}\right)+g^{+}(\mathbf{x}) .
$$

We can readily infer from $(8)$ that $\bar{h}\left(\mathbf{x} ; \mathbf{x}^{t}\right)$ is a global upper bound of $h(\mathbf{x})$ which is tight at $\mathbf{x}=\mathbf{x}^{t}$ :

$$
\bar{h}\left(\mathbf{x} ; \mathbf{x}^{t}\right) \geq h(\mathbf{x}), \text { and } \bar{h}\left(\mathbf{x}^{t} ; \mathbf{x}^{t}\right)=h\left(\mathbf{x}^{t}\right), \forall \mathbf{x} \in \mathcal{X} .
$$

In the standard MM method for problem (2) proposed in [27], a sequence of points $\left\{\mathbf{x}^{t}\right\}_{t}$ is generated by minimizing the upper bound function $\bar{h}\left(\mathbf{x} ; \mathbf{x}^{t}\right)$ :

$$
\mathbf{x}^{t+1}=\underset{\mathbf{x} \in \mathcal{X}}{\arg \min } \bar{h}\left(\mathbf{x} ; \mathbf{x}^{t}\right)
$$

This and (10) imply that $\left\{h\left(\mathbf{x}^{t}\right)\right\}_{t}$ is a decreasing sequence as

$$
h\left(\mathbf{x}^{t+1}\right) \leq \bar{h}\left(\mathbf{x}^{t+1} ; \mathbf{x}^{t}\right) \leq \bar{h}\left(\mathbf{x}^{t} ; \mathbf{x}^{t}\right)=h\left(\mathbf{x}^{t}\right) .
$$

However, the optimization problem (11) is not necessarily easy to solve due to two possible reasons: $\bar{h}\left(\mathbf{x} ; \mathbf{x}^{t}\right)$ may be nonconvex, and $\mathbf{x}^{t+1}$ may not have a closed-form expression and must be found iteratively (even when $\bar{h}\left(\mathbf{x} ; \mathbf{x}^{t}\right)$ is convex).

The proposed algorithm consists of minimizing a sequence of successively refined approximate functions. Given $\mathrm{x}^{t}$ at iteration $t$, we propose to minimize a properly designed approximate function of the upper bound function $\bar{h}\left(\mathbf{x} ; \mathbf{x}^{t}\right)$, denoted as $\tilde{h}\left(\mathbf{x} ; \mathbf{x}^{t}\right)$ :

$$
\tilde{h}\left(\mathbf{x} ; \mathbf{x}^{t}\right)=\tilde{f}\left(\mathbf{x} ; \mathbf{x}^{t}\right)-\left(\mathbf{x}-\mathbf{x}^{t}\right)^{T} \boldsymbol{\xi}^{-}\left(\mathbf{x}^{t}\right)+g^{+}(\mathbf{x}),
$$

where $\tilde{f}\left(\mathbf{x} ; \mathbf{x}^{t}\right)$ is an approximate function of $f(\mathbf{x})$ at $\mathbf{x}^{t}$ that satisfies several technical conditions that are in the same essence as those specified in [6], namely,

(A1) The approximate function $\tilde{f}\left(\mathbf{x} ; \mathbf{x}^{t}\right)$ is convex in $\mathbf{x}$ for any given $\mathbf{x}^{t} \in \mathcal{X}$

(A2) The approximate function $\tilde{f}\left(\mathbf{x} ; \mathbf{x}^{t}\right)$ is continuously differentiable in $\mathbf{x}$ for any given $\mathbf{x}^{t} \in \mathcal{X}$ and continuous in $\mathbf{x}^{t}$ for any $\mathbf{x} \in \mathcal{X}$

(A3) The gradient of $\tilde{f}\left(\mathbf{x} ; \mathbf{x}^{t}\right)$ and the gradient of $f(\mathbf{x})$ are identical at $\mathbf{x}=\mathbf{x}^{t}$ for any $\mathbf{x}^{t} \in \mathcal{X}$, i.e., $\nabla_{\mathbf{x}} \tilde{f}\left(\mathbf{x}^{t} ; \mathbf{x}^{t}\right)=\nabla_{\mathbf{x}} f\left(\mathbf{x}^{t}\right)$.

Comparing $\bar{h}\left(\mathbf{x} ; \mathbf{x}^{t}\right)$ in (9) with $\tilde{h}\left(\mathbf{x} ; \mathbf{x}^{t}\right)$ in (12), we see that replacing $f(\mathbf{x})$ in $\bar{h}\left(\mathbf{x} ; \mathbf{x}^{t}\right)$ by its approximate function $\tilde{f}\left(\mathbf{x} ; \mathbf{x}^{t}\right)$ leads to 
the proposed approximate function $\tilde{h}\left(\mathbf{x} ; \mathbf{x}^{t}\right)$. Note that $\tilde{h}\left(\mathbf{x} ; \mathbf{x}^{t}\right)$ is not necessarily a global upper bound of $\bar{h}\left(\mathbf{x} ; \mathbf{x}^{t}\right)$ (or the original function $h(\mathbf{x})$ ), because according to Assumptions (A1)-(A3), $\tilde{f}\left(\mathbf{x} ; \mathbf{x}^{t}\right)$ does not have to be a global upper bound of $f(\mathbf{x})$.

At iteration $t$, the approximate problem consists of minimizing the approximate function $\tilde{h}\left(\mathbf{x} ; \mathbf{x}^{t}\right)$ over the same constraint set $\mathcal{X}$ :

$$
\underset{\mathbf{x} \in \mathcal{X}}{\operatorname{minimize}} \underbrace{\tilde{f}\left(\mathbf{x} ; \mathbf{x}^{t}\right)-\left(\mathbf{x}-\mathbf{x}^{t}\right)^{T} \boldsymbol{\xi}^{-}\left(\mathbf{x}^{t}\right)+g^{+}(\mathbf{x})}_{\tilde{h}\left(\mathbf{x} ; \mathbf{x}^{t}\right)} .
$$

Since $\tilde{f}\left(\mathbf{x} ; \mathbf{x}^{t}\right)$ is convex by assumption (A1), (13) is a convex optimization problem. We denote as $\mathbb{B} \mathbf{x}^{t}$ an (globally) optimal solution of (13) and as $\mathcal{S}\left(\mathbf{x}^{t}\right)$ the set of (globally) optimal solutions:

$$
\mathbb{B} \mathbf{x}^{t} \in \mathcal{S}\left(\mathbf{x}^{t}\right)=\left\{\mathbf{x}^{\star}: \mathbf{x}^{\star} \in \underset{\mathbf{x} \in \mathcal{X}}{\arg \min } \tilde{h}\left(\mathbf{x} ; \mathbf{x}^{t}\right)\right\} .
$$

Based on (14), we define the mapping $\mathbb{B} \mathbf{x}$ that is used to generate the sequence of points in the proposed algorithm:

$$
\mathcal{X} \ni \mathbf{x} \longmapsto \mathbb{B} \mathbf{x} \in \mathcal{X} \text {. }
$$

Given the mapping $\mathbb{B} \mathbf{x}$, the following properties hold.

Proposition 1 (Stationary point and descent direction). Provided that Assumptions (A1)-(A3) are satisfied: (i) A point $\mathbf{x}^{t}$ is a stationary point of (2) if and only if $\mathbf{x}^{t} \in \mathcal{S}\left(\mathbf{x}^{t}\right)$ defined in (14); (ii) If $\mathbf{x}^{t}$ is not a stationary point of (14), then $\mathbb{B} \mathbf{x}^{t}-\mathrm{x}^{t}$ is a descent direction of $\bar{h}\left(\mathbf{x} ; \mathbf{x}^{t}\right)$ at $\mathbf{x}=\mathbf{x}^{t}$ in the sense that

$$
\left(\mathbb{B} \mathbf{x}^{t}-\mathbf{x}^{t}\right)^{T}\left(\nabla f\left(\mathbf{x}^{t}\right)-\boldsymbol{\xi}^{-}\left(\mathbf{x}^{t}\right)\right)+g^{+}\left(\mathbb{B} \mathbf{x}^{t}\right)-g^{+}\left(\mathbf{x}^{t}\right)<0 .
$$

Proof: See Appendix A.

If $B \mathbf{x}^{t}-\mathbf{x}^{t}$ is a descent direction of $\bar{h}\left(\mathbf{x} ; \mathbf{x}^{t}\right)$ at $\mathbf{x}=\mathbf{x}^{t}$, there exists a scalar $\gamma^{t} \in(0,1]$ such that

$$
\bar{h}\left(\mathbf{x}^{t}+\gamma^{t}\left(\mathbb{B} \mathbf{x}^{t}-\mathbf{x}^{t}\right)\right)<\bar{h}\left(\mathbf{x}^{t}\right),
$$

for which a formal proof is provided shortly in Proposition 2. This motivates us to update the variable as follows

$$
\mathbf{x}^{t+1}=\mathbf{x}^{t}+\gamma^{t}\left(\mathbb{B} \mathbf{x}^{t}-\mathbf{x}^{t}\right) .
$$

The function value $h\left(\mathbf{x}^{t}\right)$ is monotonically decreasing because

$$
h\left(\mathbf{x}^{t+1}\right) \stackrel{(a)}{\leq} \bar{h}\left(\mathbf{x}^{t+1} ; \mathbf{x}^{t}\right)<\bar{h}\left(\mathbf{x}^{t} ; \mathbf{x}^{t}\right) \stackrel{(b)}{=} h\left(\mathbf{x}^{t}\right),
$$

where $(a)$ and $(b)$ follow from (10).

There are several commonly used stepsize rules, for example, the constant/decreasing stepsize rules and the line search. In this paper, we restrict the discussion to the line search schemes because they lead to a fast convergence speed as shown in [6]. On the one hand, the traditional exact line search aims at finding the optimal stepsize, denoted as $\gamma_{\text {opt }}^{t}$ ("opt" stands for "optimal") that yields the largest decrease of $h(\mathbf{x})$ along the direction $\mathbb{B} \mathbf{x}^{t}-\mathbf{x}^{t}$ [14]:

$$
\begin{aligned}
\gamma_{\mathrm{opt}}^{t} \triangleq \underset{0 \leq \gamma \leq 1}{\arg \min } h\left(\mathbf{x}^{t}+\gamma\left(\mathbb{B} \mathbf{x}^{t}-\mathbf{x}^{t}\right)\right)-h\left(\mathbf{x}^{t}\right) \\
=\underset{0 \leq \gamma \leq 1}{\arg \min }\left\{\begin{array}{c}
f\left(\mathbf{x}^{t}+\gamma\left(\mathbb{B} \mathbf{x}^{t}-\mathbf{x}^{t}\right)\right) \\
+g^{+}\left(\mathbf{x}^{t}+\gamma\left(\mathbb{B} \mathbf{x}^{t}-\mathbf{x}^{t}\right)\right) \\
-g^{-}\left(\mathbf{x}^{t}+\gamma\left(\mathbb{B} \mathbf{x}^{t}-\mathbf{x}^{t}\right)\right)
\end{array}\right\} .
\end{aligned}
$$

Although it is a scalar problem, it is not necessarily easy to solve because it is nonconvex (even when $f(\mathbf{x})$ is convex) and nondifferentiable. On the other hand, as $\mathbb{B} \mathbf{x}^{t}-\mathbf{x}^{t}$ is also a descent direction of $\bar{h}\left(\mathbf{x} ; \mathbf{x}^{t}\right)$ according to Proposition 1 , it is possible to perform the exact line search over the upper bound function $\bar{h}\left(\mathbf{x} ; \mathbf{x}^{t}\right)$ along the direction $\mathbb{B} \mathbf{x}^{t}-\mathbf{x}^{t}$ :

$$
\begin{aligned}
\gamma_{\mathrm{ub}}^{t} \triangleq \underset{0 \leq \gamma \leq 1}{\arg \min } \bar{h}\left(\mathbf{x}^{t}+\gamma\left(\mathbb{B} \mathbf{x}^{t}-\mathbf{x}^{t}\right) ; \mathbf{x}^{t}\right)-\bar{h}\left(\mathbf{x}^{t} ; \mathbf{x}^{t}\right) \\
=\underset{0 \leq \gamma \leq 1}{\arg \min }\left\{\begin{array}{l}
f\left(\mathbf{x}^{t}+\gamma\left(\mathbb{B} \mathbf{x}^{t}-\mathbf{x}^{t}\right)\right) \\
-\left(\mathbf{x}^{t}+\gamma\left(\mathbb{B} \mathbf{x}^{t}-\mathbf{x}^{t}\right)-\mathbf{x}^{t}\right)^{T} \boldsymbol{\xi}^{-}\left(\mathbf{x}^{t}\right) \\
g^{+}\left(\mathbf{x}^{t}+\gamma\left(\mathbb{B} \mathbf{x}^{t}-\mathbf{x}^{t}\right)\right)
\end{array}\right\},
\end{aligned}
$$

and we denote as $\gamma_{\text {ub }}^{t}$ ("ub" stands for "upper bound") the obtained stepsize. However, this is not always favorable in practice either because the above minimization problem involves the nonsmooth function $g^{+}$.

To reduce the complexity of traditional exact line search schemes in (19), we start from (19b): applying the Jensen's inequality to the convex function $g^{+}$in (19b) yields that for any $\gamma \in[0,1]$,

$$
\begin{aligned}
g^{+}\left(\mathbf{x}^{t}+\gamma\left(\mathbb{B} \mathbf{x}^{t}-\mathbf{x}^{t}\right)\right) & \leq\left(1-\gamma^{t}\right) g^{+}\left(\mathbf{x}^{t}\right)+\gamma g^{+}\left(\mathbb{B} \mathbf{x}^{t}\right) \\
& =g^{+}\left(\mathbf{x}^{t}\right)+\gamma\left(g^{+}\left(\mathbb{B} \mathbf{x}^{t}\right)-g^{+}\left(\mathbf{x}^{t}\right)\right) .
\end{aligned}
$$

The function on the right hand side of (20) is a differentiable and linear function in $\gamma$. We thus propose to perform the line search over the following function which is obtained by replacing the nonsmooth function $g^{+}$in (19b) by its upper bound (20):

$$
\begin{aligned}
\gamma^{t}=\underset{0 \leq \gamma \leq 1}{\arg \min }\left\{\begin{array}{c}
f\left(\mathbf{x}^{t}+\gamma\left(\mathbb{B} \mathbf{x}^{t}-\mathbf{x}^{t}\right)\right) \\
-\left(\mathbf{x}^{t}+\gamma\left(\mathbb{B} \mathbf{x}^{t}-\mathbf{x}^{t}\right)-\mathbf{x}^{t}\right)^{T} \boldsymbol{\xi}^{-}\left(\mathbf{x}^{t}\right) \\
+g^{+}\left(\mathbf{x}^{t}\right)+\gamma\left(g^{+}\left(\mathbb{B} \mathbf{x}^{t}\right)-g^{+}\left(\mathbf{x}^{t}\right)\right) .
\end{array}\right\} \\
=\underset{0 \leq \gamma \leq 1}{\arg \min }\left\{\begin{array}{c}
f\left(\mathbf{x}^{t}+\gamma\left(\mathbb{B} \mathbf{x}^{t}-\mathbf{x}^{t}\right)\right) \\
+\gamma\left(g^{+}\left(\mathbb{B} \mathbf{x}^{t}\right)-g^{+}\left(\mathbf{x}^{t}\right)-\left(\mathbb{B} \mathbf{x}^{t}-\mathbf{x}^{t}\right)^{T} \boldsymbol{\xi}^{-}\left(\mathbf{x}^{t}\right)\right)
\end{array}\right\} .
\end{aligned}
$$

Combining (19b) and (20), we readily see that the function in (19b) is upper bounded by the function in (21) which is tight at $\gamma=0$. The optimization problem in (21) is differentiable and presumably much easier to optimize than the nondifferentiable problems in (19). It is furthermore convex if $f(\mathbf{x})$ is convex, and it can be solved efficiently by the bisection method; in many cases closed-form expressions exist, as we will show later by the example applications in Sec. IV-V. This is a desirable property because the scalar optimization problem in (21) is convex as long as $f$ is convex, although the original function $h$ is still not convex due to $g^{-}$.

Albeit the low complexity, a natural question to ask is whether the stepsize $\gamma^{t}$ obtained by the proposed exact line search scheme (21) leads to a strict decrease of the original objective function $h(\mathbf{x}){ }^{2}$ The answer is affirmative and we first provide an intuitive explanation: the gradient of the function in (21) w.r.t. $\gamma$ at $\gamma=0$ is

$$
\left(\mathbb{B} \mathbf{x}^{t}-\mathbf{x}^{t}\right)^{T} \nabla f\left(\mathbf{x}^{t}\right)+g^{+}\left(\mathbb{B} \mathbf{x}^{t}\right)-g^{+}\left(\mathbf{x}^{t}\right)-\left(\mathbb{B} \mathbf{x}^{t}-\mathbf{x}^{t}\right)^{T} \boldsymbol{\xi}^{-}\left(\mathbf{x}^{t}\right),
$$

which is strictly smaller than 0 according to Proposition 1 . This implies the function has a negative slope at $\gamma=0$ and its minimum point $\gamma^{t}$ is thus nonzero and positive. Consequently the objective function $h(\mathbf{x})$ can be strictly decreased: $h\left(\mathbf{x}^{t}+\gamma^{t}\left(\mathbb{B} \mathbf{x}^{t}-\mathbf{x}^{t}\right)\right)<h\left(\mathbf{x}^{t}\right)$. This intuitive explanation will be made rigorous shortly in Proposition 2 .

If no structure in $f(\mathbf{x})$ (e.g., convexity) can be exploited to efficiently compute $\gamma^{t}$ that minimizes the objective function in (21)

${ }^{2}$ With a slight abuse of terminology, we call the proposed line search scheme (21) the exact line search, although it is carried out over a differentiable upper bound of the original objective function $h$. 


$$
\begin{aligned}
& f\left(\mathbf{x}^{t}+\beta^{m}\left(\mathbb{B} \mathbf{x}^{t}-\mathbf{x}^{t}\right)\right)-\left(\mathbf{x}^{t}+\beta^{m}\left(\mathbb{B} \mathbf{x}^{t}-\mathbf{x}^{t}\right)-\mathbf{x}^{t}\right)^{T} \boldsymbol{\xi}^{-}\left(\mathbf{x}^{t}\right)+g^{+}\left(\mathbf{x}^{t}\right)+\beta^{m}\left(g^{+}\left(\mathbb{B} \mathbf{x}^{t}\right)-g^{+}\left(\mathbf{x}^{t}\right)\right) \\
\leq & f\left(\mathbf{x}^{t}\right)+g^{+}\left(\mathbf{x}^{t}\right)+\alpha \beta^{m}\left(\left(\mathbb{B} \mathbf{x}^{t}-\mathbf{x}^{t}\right)^{T}\left(\nabla f\left(\mathbf{x}^{t}\right)-\boldsymbol{\xi}^{-}\left(\mathbf{x}^{t}\right)\right)+g^{+}\left(\mathbb{B} \mathbf{x}^{t}\right)-g^{+}\left(\mathbf{x}^{t}\right)\right) . \\
& f\left(\mathbf{x}^{t}+\beta^{m}\left(\mathbb{B} \mathbf{x}^{t}-\mathbf{x}^{t}\right)\right)-\beta^{m}\left(\mathbb{B} \mathbf{x}^{t}-\mathbf{x}^{t}\right)^{T} \boldsymbol{\xi}^{-}\left(\mathbf{x}^{t}\right)+\beta^{m}\left(g^{+}\left(\mathbb{B} \mathbf{x}^{t}\right)-g^{+}\left(\mathbf{x}^{t}\right)\right) \\
\leq & f\left(\mathbf{x}^{t}\right)+\alpha \beta^{m}\left(\left(\mathbb{B} \mathbf{x}^{t}-\mathbf{x}^{t}\right)^{T}\left(\nabla f\left(\mathbf{x}^{t}\right)-\boldsymbol{\xi}^{-}\left(\mathbf{x}^{t}\right)\right)+g^{+}\left(\mathbb{B} \mathbf{x}^{t}\right)-g^{+}\left(\mathbf{x}^{t}\right)\right) .
\end{aligned}
$$

\author{
Algorithm 1 The proposed successive convex approximation frame- \\ work for problem (2) \\ Data: $t=0, \mathbf{x}^{0}$ (arbitrary but fixed, e.g., $\mathbf{x}^{0}=\mathbf{0}$ ), stop criterion $\delta$. \\ S1: Compute $\mathbb{B x}^{t}$ according to (14). \\ S2: Determine the stepsize $\gamma^{t}$ by the exact line search (21) or the \\ successive line search (23). \\ S3: Update $\mathrm{x}^{t+1}$ according to (17). \\ S4: If $\left|\left(\mathbb{B} \mathbf{x}^{t}-\mathbf{x}^{t}\right)^{T}\left(\nabla f\left(\mathbf{x}^{t}\right)-\boldsymbol{\xi}^{-}\left(\mathbf{x}^{t}\right)\right)+g^{+}\left(\mathbb{B} \mathbf{x}^{t}\right)-g^{+}\left(\mathbf{x}^{t}\right)\right| \leq \delta$, \\ STOP; otherwise $t \leftarrow t+1$ and go to $\mathbf{S 1}$.
}

exactly, we adopt a stepsize if it yields sufficient decrease in the sense specified by the successive line search (also known as the Armijo rule) [17]. In particular, the successive line search counterpart of (19a) or (19b) is as follows: given scalars $0<\alpha<1$ and $0<\beta<1$, the stepsize $\gamma^{t}$ is set to be $\gamma^{t}=\beta^{m_{t}}$, where $m_{t}$ is the smallest nonnegative integer $m$ satisfying the following inequality:

$$
\begin{aligned}
& h\left(\mathbf{x}^{t}+\beta^{m}\left(\mathbb{B} \mathbf{x}^{t}-\mathbf{x}^{t}\right)\right)-h\left(\mathbf{x}^{t}\right) \\
& \quad \leq \alpha \beta^{m}\left(\left(\mathbb{B} \mathbf{x}^{t}-\mathbf{x}^{t}\right)^{T}\left(\nabla f\left(\mathbf{x}^{t}\right)-\boldsymbol{\xi}^{-}\left(\mathbf{x}^{t}\right)\right)+g^{+}\left(\mathbb{B} \mathbf{x}^{t}\right)-g^{+}\left(\mathbf{x}^{t}\right)\right),
\end{aligned}
$$

or

$$
\begin{aligned}
& \bar{h}\left(\mathbf{x}^{t}+\beta^{m}\left(\mathbb{B} \mathbf{x}^{t}-\mathbf{x}^{t}\right)\right)-\bar{h}\left(\mathbf{x}^{t} ; \mathbf{x}^{t}\right) \\
& \quad \leq \alpha \beta^{m}\left(\left(\mathbb{B} \mathbf{x}^{t}-\mathbf{x}^{t}\right)^{T}\left(\nabla f\left(\mathbf{x}^{t}\right)-\boldsymbol{\xi}^{-}\left(\mathbf{x}^{t}\right)\right)+g^{+}\left(\mathbb{B} \mathbf{x}^{t}\right)-g^{+}\left(\mathbf{x}^{t}\right)\right),
\end{aligned}
$$

As a result, $g^{+}\left(\mathbf{x}^{t}+\beta^{m}\left(\mathbb{B} \mathbf{x}^{t}-\mathbf{x}^{t}\right)\right)$ (in $h$ or $\bar{h}$ ) must be evaluated for $m_{t}+1$ times, namely, $m=0,1, \ldots, m_{t}$, and this may incur a high complexity.

To reduce the complexity of traditional successive line search schemes (22), we follow the reasoning from (19) to (21) and propose a successive line search that works as follows (the detailed derivation steps are deferred to Appendix A): given scalars $0<\alpha<1$ and $0<\beta<1$, the stepsize $\gamma^{t}$ is set to be $\gamma^{t}=\beta^{m_{t}}$, where $m_{t}$ is the smallest nonnegative integer $m$ satisfying the inequality in (23) shown at the top of this page, which is the same as (24) after removing the constants that appear on both sides. Note that the smooth function $f$ needs to be evaluated several times for $m=1,2, \ldots, m_{t}$ as in traditional successive line search scheme, but we only have to evaluate the nonsmooth function $g^{+}$once at $\mathbb{B} \mathbf{x}^{t}$, i.e., $g^{+}\left(\mathbb{B} \mathbf{x}^{t}\right)$.

We show in the following proposition that the stepsize obtained by the proposed exact/successive line search (21) and (23) is nonzero, i.e., $\gamma^{t} \in(0,1]$ and $h\left(\mathbf{x}^{t+1}\right)<h\left(\mathbf{x}^{t}\right)$.

Proposition 2 (Existence of a nontrivial stepsize). If $\mathbb{B} \mathbf{x}^{t}-\mathbf{x}^{t}$ is a descent direction of $\bar{h}\left(\mathbf{x} ; \mathbf{x}^{t}\right)$ at the point $\mathbf{x}=\mathbf{x}^{t}$ in the sense of (16), then the stepsize given by the proposed exact line search (21) or the proposed successive line search (23) is nonzero, i.e., $\gamma^{t} \in(0,1]$.

Proof: See Appendix A.

The proposed SCA framework is summarized in Algorithm 1 and its convergence properties are given in the following theorem.
Theorem 3 (Convergence to a stationary point). Consider the sequence $\left\{\mathrm{x}^{t}\right\}$ generated by Algorithm 1. Provided that Assumptions (A1)-(A3) as well as the following assumptions are satisfied:

(A4) The solution set $\mathcal{S}\left(\mathbf{x}^{t}\right)$ is nonempty for $t=1,2, \ldots$;

(A5) Given any convergent subsequence $\left\{\mathbf{x}^{t}\right\}_{t \in \mathcal{T}}$ where $\mathcal{T} \subseteq$ $\{1,2, \ldots\}$, the sequence $\left\{\mathbb{B}^{t}\right\}_{t \in \mathcal{T}}$ is bounded.

Then any limit point of $\left\{\mathbf{x}^{t}\right\}$ is a stationary point of (2).

Proof: See Appendix B.

Sufficient conditions for Assumptions (A4)-(A5) are that either the feasible set $\mathcal{X}$ in (13) is bounded or the approximate function in (13) is strongly convex [37]. We will show that these assumptions are satisfied by the example application in the next section.

If the approximate function $\tilde{f}\left(\mathbf{x} ; \mathbf{x}^{t}\right)$ in (12) satisfies an additional assumption that it is a global upper bound of $f(\mathbf{x})$, i.e., assume that (A6) $\tilde{f}\left(\mathbf{x} ; \mathbf{x}^{t}\right) \geq f(\mathbf{x})$ and $\tilde{f}\left(\mathbf{x}^{t} ; \mathbf{x}^{t}\right)=f\left(\mathbf{x}^{t}\right)$,

then the proposed Algorithm 1 converges (in the sense specified by Theorem 3) under a constant unit stepsize $\gamma^{t}=1$, as it yields a larger decrease than the successive line search. We refer the interested reader to [6, Eqs. (15)-(17)] for more details.

In what follows, we draw some comments on the proposed algorithm's features and connections to existing algorithms.

On the choice of approximate function. Note that different choices of $\tilde{f}\left(\mathbf{x} ; \mathbf{x}^{t}\right)$ lead to different algorithms. We mention for the self-containedness of this paper two commonly used approximate functions, and assume for now that the constraint set $\mathcal{X}$ has a Cartesian product structure and $g^{+}$is separable, i.e., $g^{+}(\mathbf{x})=$ $\sum_{k=1}^{K} g^{+}\left(\mathbf{x}_{k}\right)$. We refer the interested readers to [6, Sec. III-B] for a more comprehensive discussion.

Proximal type approximation. The proximal type approximate function $\tilde{h}\left(\mathbf{x} ; \mathbf{x}^{t}\right)$ has the following form [5, Sec. 4.2]:

$\underbrace{f\left(\mathbf{x}^{t}\right)+\nabla f\left(\mathbf{x}^{t}\right)\left(\mathbf{x}-\mathbf{x}^{t}\right)+\frac{c^{t}}{2}\left\|\mathbf{x}-\mathbf{x}^{t}\right\|^{2}}_{\tilde{f}\left(\mathbf{x} ; \mathbf{x}^{t}\right)}-\left(\mathbf{x}-\mathbf{x}^{t}\right)^{T} \boldsymbol{\xi}^{-}\left(\mathbf{x}^{t}\right)+g(\mathbf{x})$

where $c^{t}>0$. Since the approximate function is separable among the different block variables and the constraint set has a Cartesian structure, minimizing the approximate function to obtain $\mathbb{B} \mathbf{x}^{t}$ is equivalent to set $\mathbb{B} \mathbf{x}^{t}=\left(\mathbb{B}_{k} \mathbf{x}^{t}\right)_{k=1}^{K}$ where $\mathbf{x}=\left(\mathbf{x}_{k}\right)_{k=1}^{K}$ and

$$
\mathbb{B}_{k} \mathbf{x}^{t} \triangleq \underset{\mathbf{x}_{k} \in \mathcal{X}_{k}}{\arg \min }\left\{\begin{array}{l}
\nabla_{k} f\left(\mathbf{x}^{t}\right)\left(\mathbf{x}_{k}-\mathbf{x}_{k}^{t}\right)+\frac{c^{t}}{2}\left\|\mathbf{x}_{k}-\mathbf{x}_{k}^{t}\right\|^{2} \\
-\left(\mathbf{x}_{k}-\mathbf{x}_{k}^{t}\right)^{T} \boldsymbol{\xi}_{k}^{-}\left(\mathbf{x}^{t}\right)+g^{+}\left(\mathbf{x}_{k}\right)
\end{array}\right\},
$$

for all $k=1, \ldots, K$. According to Theorem 3 and the discussion that immediately follows, the proposed algorithm converges under a constant unit stepsize if $\tilde{f}\left(\mathbf{x} ; \mathbf{x}^{t}\right)$ in (25a) is a global upper bound of $f(\mathbf{x})$, which is indeed the case when $c^{t} \geq L_{\nabla f}\left(L_{\nabla f}\right.$ is the Lipschitz constant of $\nabla f$ ) in view of the descent lemma [17, Prop. A.24].

Best-response type approximation. In problem (2), if $f(\mathbf{x})$ is convex in each $\mathbf{x}_{k}$ where $k=1, \ldots, K$ (but not necessarily jointly convex in $\left.\left(\mathbf{x}_{1}, \ldots, \mathbf{x}_{K}\right)\right)$, the best-response type approximate 


\begin{tabular}{|c|c|c|c|c|c|}
\hline Algorithm & $f$ & $g^{+}$ & $g^{-}$ & The approximate function $\tilde{f}$ in (13) & The stepsize $\gamma^{t}$ in variable update (17) \\
\hline The proposed SCA & nonconvex & convex & convex & (A1)-(A3) & line search (21) or (24), $\gamma^{t}=1$ if (A6) \\
\hline MM [27] & convex & convex & convex & $\tilde{f}\left(\mathbf{x} ; \mathbf{x}^{t}\right)=f(\mathbf{x})$ & $\gamma^{t}=1$ \\
\hline SCA [6] & nonconvex & convex & 0 & $(\mathrm{~A} 1)-(\mathrm{A} 3)$ & line search (21) or (24), $\gamma^{t}=1$ if (A6) \\
\hline GIST [7] & nonconvex & convex & 0 & $\tilde{f}\left(\mathbf{x} ; \mathbf{x}^{t}\right)=\left(\mathbf{x}-\mathbf{x}^{t}\right)^{T} \nabla f\left(\mathbf{x}^{t}\right)+\frac{c^{t}}{2}\left\|\mathbf{x}-\mathbf{x}^{t}\right\|^{2}$ & $\gamma^{t}=1$ if $c^{t} \geq L_{\nabla f}$ \\
\hline
\end{tabular}

Table I

CONNECTION BETWEEN THE PROPOSED SCA FRAMEWORK AND EXISTING ALGORITHMS IN TERMS OF THE ASSUMPTIONS ON $f, g^{+}, g^{-}, \tilde{f}$ AND $\gamma^{t}$.

function is defined as

$$
\tilde{f}\left(\mathbf{x} ; \mathbf{x}^{t}\right)=\sum_{k=1}^{K} f\left(\mathbf{x}_{k}, \mathbf{x}_{-k}^{t}\right),
$$

and the approximate problem is

$$
\mathbb{B}_{k} \mathbf{x}^{t}=\underset{\mathbf{x}_{k} \in \mathcal{X}_{k}}{\arg \min }\left\{f\left(\mathbf{x}_{k}, \mathbf{x}_{-k}^{t}\right)-\left(\mathbf{x}_{k}-\mathbf{x}_{k}^{t}\right)^{T} \boldsymbol{\xi}_{k}^{-}\left(\mathbf{x}^{t}\right)+g\left(\mathbf{x}_{k}\right)\right\}
$$

for all $k=1, \ldots, K$. Comparing (26) with (25), we see that the function $f$ is not linearized in (26b). The best-response type algorithm typically converges faster than the proximal type algorithm because the desirable property such as convexity is preserved in the bestresponse type approximation while it is lost when $f(\mathbf{x})$ is being linearized in the proximal type approximation.

On the proposed line search schemes. Since the objective function in the proposed exact line search scheme (21) is an upper bound of the objective function in (19b) (see the discussion after (21)), the obtained decrease by the proposed line search $\gamma^{t}$ in (21) is generally smaller than that of $\gamma_{\mathrm{ub}}^{t}$ in (19b), the line search over the upper bound function $\bar{h}\left(\mathbf{x} ; \mathbf{x}^{t}\right)$, which is furthermore smaller than that of $\gamma_{\text {opt }}^{t}$ in (19a), the line search over the original function $h(\mathbf{x})$ :

$$
\begin{aligned}
h\left(\mathbf{x}^{t}+\gamma_{\mathrm{opt}}^{t}\left(\mathbb{B} \mathbf{x}^{t}-\mathbf{x}^{t}\right)\right) & \leq h\left(\mathbf{x}^{t}+\gamma_{\mathrm{ub}}^{t}\left(\mathbb{B} \mathbf{x}^{t}-\mathbf{x}^{t}\right)\right) \\
& \leq h\left(\mathbf{x}^{t}+\gamma^{t}\left(\mathbb{B} \mathbf{x}^{t}-\mathbf{x}^{t}\right)\right)<h\left(\mathbf{x}^{t}\right) .
\end{aligned}
$$

Nevertheless, the order of the complexity performing the line search is reversed. To see this, assume $f$ is convex. Then the optimization problem computing $\gamma_{\mathrm{opt}}^{t}$ in (19a), $\gamma_{\mathrm{ub}}^{t}$ in (19b), and $\gamma^{t}$ in (21) is nonconvex and nondifferentiable, convex but nondifferentiable, and convex and differentiable, respectively. Therefore, the proposed line search scheme achieves a good tradeoff between performance and complexity, and this will be illustrated by the example applications in Sec. IV-V.

On the convergence of the proposed algorithm. The proposed algorithm presents a fast convergence behavior because we could choose the approximate function so that the problem structure is exploited to a larger extent, for example, the partial convexity in the best-response type approximation. Besides, the line search leads to a much faster convergence than predetermined stepsizes such as constant stepsizes and decreasing stepsizes. Furthermore, the proposed algorithm converges even when $\nabla f$ is not Lipschitz continuous, and is thus applicable for a wider class of problems.

On the complexity of the proposed algorithm. The Algorithm 1 has a low complexity due to the use of an approximate function and the line search scheme over a differentiable function. The benefits of employing the approximate function $\tilde{f}\left(\mathbf{x} ; \mathbf{x}^{t}\right)$ are twofold. On the one hand, it is a convex function by Assumption (A1), so the approximate problem (13) is a convex problem, which is presumably easier to solve than (11) which is nonconvex if $f(\mathbf{x})$ is nonconvex On the other hand, it can be tailored according to the structure of the problem at hand so that the approximate problem (13) is even easier to solve. For example, if $g^{+}(\mathbf{x})$ is separable among the scalar elements of $\mathbf{x}$ (as in, e.g., $\ell_{1}$-norm $\|\mathbf{x}\|_{1}=\sum_{k=1}^{K}\left|x_{k}\right|$ ), we can choose $\tilde{f}\left(\mathbf{x} ; \mathbf{x}^{t}\right)$ to be separable as well, so that the problem (13) can be decomposed into independent subproblems which are then solved in parallel. Furthermore, the proposed line search scheme (21) is carried out over a differentiable function, which is presumably much easier to implement than traditional schemes (19) over nonconvex nonsmooth functions.

On the connection to the classic MM method [27]. Assume $f$ is convex. $^{3}$ The proposed algorithm includes as a special case the MM method proposed in [27] by setting $\tilde{f}\left(\mathbf{x} ; \mathbf{x}^{t}\right)=f(\mathbf{x})$ and $\gamma^{t}=1$. For this particular choice of approximate function, it can be verified that the assumptions (A1)-(A3) are satisfied. Furthermore, $\tilde{f}\left(\mathbf{x} ; \mathbf{x}^{t}\right)=$ $f(\mathbf{x})$ is a trivial upper bound of $f(\mathbf{x})$ and Assumption (A6) is thus satisfied. We can therefore skip the line search procedure and directly choose the constant unit stepsize $\gamma^{t}=1$ for all $t$. Interpreting the MM method as a special case of the proposed algorithm consolidates once more that choosing a possibly different approximate function $\tilde{f}\left(\mathbf{x} ; \mathbf{x}^{t}\right)$ that leads to an easier optimization problem at each iteration than the original function $f(\mathbf{x})$ as in the classic MM method may notably reduce the algorithm's complexity.

On the connection to the SCA framework for a convex $g$ [6]. The proposed framework includes as a special case the SCA framework proposed in [6] for a convex $g$ : assume $g^{-}(\mathbf{x})=0$, and the approximate function in (12) reduces to $\tilde{h}\left(\mathbf{x} ; \mathbf{x}^{t}\right)=\tilde{f}\left(\mathbf{x} ; \mathbf{x}^{t}\right)+g^{+}(\mathbf{x})$.

On the connection to the GIST algorithm [7]. Assume $g^{-}(\mathbf{x})=$ $0 .^{4}$ In the GIST algorithm [38], the variable is updated as follows:

$$
\mathbf{x}^{t+1}=\underset{\mathbf{x}}{\arg \min }\left(\mathbf{x}-\mathbf{x}^{t}\right) \nabla f\left(\mathbf{x}^{t}\right)+\frac{c^{t}}{2}\left\|\mathbf{x}-\mathbf{x}^{t}\right\|^{2}+g^{+}(\mathbf{x}) .
$$

This is a special case of the proximal type algorithm by choosing $c^{t} \geq L_{\nabla f}$ and $\gamma^{t}=1$. When the value of $L_{\nabla f}$ is unknown, $c^{t}$ is estimated iteratively [29]: for a constant $\beta \in(0,1)$, define $\mathbf{x}^{\star}\left(\beta^{m}\right)$ as

$$
\mathbf{x}^{\star}\left(\beta^{m}\right) \triangleq \underset{\mathbf{x}}{\arg \min }\left(\mathbf{x}-\mathbf{x}^{t}\right) \nabla f\left(\mathbf{x}^{t}\right)+\frac{1}{2 \beta^{m}}\left\|\mathbf{x}-\mathbf{x}^{t}\right\|^{2}+g^{+}(\mathbf{x}) .
$$

Then $c^{t}=1 / \beta^{m_{t}}$ and $\mathbf{x}^{t+1}=\mathbf{x}^{\star}\left(\beta^{m_{t}}\right)$ while $m_{t}$ is the smallest nonnegative integer such that the following inequality is satisfied for some $\alpha \in(0,1)$ :

$$
\begin{aligned}
& f\left(\mathbf{x}^{\star}\left(\eta^{m_{t}}\right)\right)+g\left(\mathbf{x}^{\star}\left(\eta^{m_{t}}\right)\right) \\
< & f\left(\mathbf{x}^{t}\right)+g\left(\mathbf{x}^{t}\right)-\frac{\alpha}{2 \beta^{m_{t}}}\left\|\mathbf{x}^{\star}\left(\eta^{m_{t}}\right)-\mathbf{x}^{t}\right\|^{2} .
\end{aligned}
$$

This implies that, in the GIST algorithm, $\mathbf{x}^{\star}\left(\beta^{m}\right)$ and $g\left(\mathbf{x}^{\star}\left(\beta^{m}\right)\right)$ are evaluated for $m_{t}+1$ times, namely, $m=0,1, \ldots, m_{t}$. This is however not necessary in the proposed successive line search (23), because $\mathbb{B} \mathbf{x}^{t}$ given by (14) does not depend on any unknown parameters and both $\mathbb{B} \mathbf{x}^{t}$ and $g^{+}\left(\mathbb{B}^{t}\right)$ only need to be computed once. Therefore, the algorithmic complexity could be notably reduced by employing a convex approximate function that is not necessarily an upper bound of the original function $h(\mathbf{x})$.

${ }^{3}$ This is an assumption made in [27].

${ }^{4}$ This is an assumption made in [38]. 
The above discussion on the connection to existing algorithms is summarized in Table I.

\section{Network Anomaly Detection Through Sparsity REGULARIZED RANK MINIMIZATION}

In this section, we propose an iterative algorithm by customizing Algorithm 1 to solve the network anomaly detection problem introduced in Sec. II-A.

\section{A. Network anomaly detection based on matrix factorization}

For the simplicity of cross reference, we first duplicate the problem formulation (3) here:

$$
\underset{\mathbf{P}, \mathbf{Q}, \mathbf{S}}{\operatorname{minimize}} \frac{1}{2}\|\mathbf{P Q}+\mathbf{D S}-\mathbf{Y}\|_{F}^{2}+\frac{\lambda}{2}\left(\|\mathbf{P}\|_{F}^{2}+\|\mathbf{Q}\|_{F}^{2}\right)+\mu\|\mathbf{S}\|_{1},
$$

and remark again that problem (29) is a special case of (2) by setting

$$
\begin{aligned}
f(\mathbf{P}, \mathbf{Q}, \mathbf{S}) & \triangleq \frac{1}{2}\|\mathbf{P Q}+\mathbf{D S}-\mathbf{Y}\|_{F}^{2}+\frac{\lambda}{2}\left(\|\mathbf{P}\|_{F}^{2}+\|\mathbf{Q}\|_{F}^{2}\right), \\
g(\mathbf{S}) & \triangleq \mu\|\mathbf{S}\|_{1},
\end{aligned}
$$

where $g(\mathbf{S})$ is convex. To simplify the notation, we use $\mathbf{Z}$ as a compact notation for $(\mathbf{P}, \mathbf{Q}, \mathbf{S}): \mathbf{Z} \triangleq(\mathbf{P}, \mathbf{Q}, \mathbf{S})$; in the rest of this section, $\mathbf{Z}$ and $(\mathbf{P}, \mathbf{Q}, \mathbf{S})$ are used interchangeably.

Related work. We first briefly describe the BCD algorithm adopted in [12] to find a stationary point of the nonconvex problem (29), where the variables are updated sequentially according to their bestresponse. For example, when $\mathbf{P}$ (or $\mathbf{Q}$ ) is updated, the variables $(\mathbf{Q}, \mathbf{S})$ (or $(\mathbf{P}, \mathbf{S})$ ) are fixed. When $(\mathbf{P}, \mathbf{Q})$ is fixed for example, the optimization problem w.r.t. $\mathbf{S}$ decouples among its columns:

$$
\begin{aligned}
& \frac{1}{2}\|\mathbf{P Q}+\mathbf{D S}-\mathbf{Y}\|_{F}^{2}+\mu\|\mathbf{S}\|_{1} \\
= & \sum_{k=1}^{K}\left(\frac{1}{2}\left\|\mathbf{P} \mathbf{q}_{k}-\mathbf{D} \mathbf{s}_{k}-\mathbf{y}_{k}\right\|_{2}^{2}+\mu\left\|\mathbf{s}_{k}\right\|_{1}\right),
\end{aligned}
$$

where $\mathbf{q}_{k}, \mathbf{s}_{k}$ and $\mathbf{y}_{k}$ is the $k$-th column of $\mathbf{Q}, \mathbf{S}$ and $\mathbf{Y}$, respectively However, the optimization problem w.r.t. $\mathbf{s}_{k}$ does not have a closedform solution and is not easy to solve. To reduce the complexity, the elements of $\mathbf{S}$ are updated row-wise, as the optimization problem w.r.t. $s_{i, k}$, the $(i, k)$-th element of $\mathbf{S}$, has a closed-form solution:

$\underset{\left(s_{i, k}\right)_{k=1}^{K}}{\operatorname{minimize}} \sum_{k=1}^{K}\left(\begin{array}{l}\frac{1}{2}\left\|\mathbf{P q}_{k}-\mathbf{d}_{i} s_{i, k}-\sum_{j=1, j \neq i}^{I} \mathbf{d}_{j} s_{j, k}-\mathbf{y}_{k}\right\|_{2}^{2} \\ +\mu\left|s_{i, k}\right|+\mu \sum_{j=1, j \neq i}^{K}\left|s_{j, k}\right|\end{array}\right)$

where $\mathbf{d}_{i}$ is the $i$-th column of $\mathbf{D}$, and $s_{i, k}$ is the $i$-th element of $\mathbf{s}_{k}$ (and hence the $(j, k)$-th element of $\mathbf{S}$ ). Solving the above optimization problem w.r.t. $\left(s_{i, k}\right)_{k=1}^{K}$ for a given $i$ results in simultaneous update of all elements in the same ( $i$-th) row of $\mathbf{S}$, and changing $i$ from iteration to iteration results in the sequential row-wise update. Nevertheless, a major drawback of the sequential row-wise update is that it may incur a large delay because the $(i+1)$-th row cannot be updated until the $i$-th row is updated and the delay may be very large when $I$, the number of rows, is large, which is a norm rather than an exception in big data analytics [13].

Proposed algorithm. Although $f(\mathbf{P}, \mathbf{Q}, \mathbf{S})$ in (29) is not jointly convex w.r.t. $(\mathbf{P}, \mathbf{Q}, \mathbf{S})$, it is individual convex in $\mathbf{P}, \mathbf{Q}$ and $\mathbf{S}$. In other words, $f(\mathbf{P}, \mathbf{Q}, \mathbf{S})$ is convex w.r.t. one variable while the other two variables are fixed. This leads to the best-response type approximation: given $\mathbf{Z}^{t}=\left(\mathbf{P}^{t}, \mathbf{Q}^{t}, \mathbf{S}^{t}\right)$ in iteration $t$, we approximate the original nonconvex function $f(\mathbf{Z})$ by a convex function $\tilde{f}\left(\mathbf{Z} ; \mathbf{Z}^{t}\right)$ that is of the following form,

$$
\tilde{f}\left(\mathbf{Z} ; \mathbf{Z}^{t}\right)=\tilde{f}_{P}\left(\mathbf{P} ; \mathbf{Z}^{t}\right)+\tilde{f}_{Q}\left(\mathbf{Q} ; \mathbf{Z}^{t}\right)+\tilde{f}_{S}\left(\mathbf{S} ; \mathbf{Z}^{t}\right),
$$

where

$$
\begin{aligned}
\tilde{f}_{P}\left(\mathbf{P} ; \mathbf{Z}^{t}\right) \triangleq & f\left(\mathbf{P}, \mathbf{Q}^{t}, \mathbf{S}^{t}\right)=\frac{1}{2}\left\|\mathbf{P} \mathbf{Q}^{t}+\mathbf{D} \mathbf{S}^{t}-\mathbf{Y}\right\|_{F}^{2}+\frac{\lambda}{2}\|\mathbf{P}\|_{F}^{2}, \\
\tilde{f}_{Q}\left(\mathbf{Q} ; \mathbf{Z}^{t}\right) \triangleq & f\left(\mathbf{P}^{t}, \mathbf{Q}, \mathbf{S}^{t}\right)=\frac{1}{2}\left\|\mathbf{P}^{t} \mathbf{Q}+\mathbf{D} \mathbf{S}^{t}-\mathbf{Y}\right\|_{F}^{2}+\frac{\lambda}{2}\|\mathbf{Q}\|_{F}^{2}, \\
\tilde{f}_{S}\left(\mathbf{S} ; \mathbf{Z}^{t}\right) \triangleq & \sum_{i, k} f\left(\mathbf{P}^{t}, \mathbf{Q}^{t}, s_{i, k},\left(s_{j, k}^{t}\right)_{j \neq i},\left(\mathbf{s}_{j}^{t}\right)_{j \neq i}\right) \\
= & \sum_{i, k} \frac{1}{2}\left\|\mathbf{P}^{t} \mathbf{q}_{k}^{t}+\mathbf{d}_{i} s_{i, k}+\sum_{j \neq i} \mathbf{d}_{j} s_{j, k}^{t}-\mathbf{y}_{k}\right\|_{2}^{2} \\
= & \operatorname{tr}\left(\mathbf{S}^{T} \operatorname{diag}\left(\mathbf{D}^{T} \mathbf{D}\right) \mathbf{S}\right) \\
& -\operatorname{tr}\left(\mathbf{S}^{T}\left(\operatorname{diag}\left(\mathbf{D}^{T} \mathbf{D}\right) \mathbf{S}^{t}-\mathbf{D}^{T}\left(\mathbf{D} \mathbf{S}^{t}-\mathbf{Y}+\mathbf{P}^{t} \mathbf{Q}^{t}\right)\right)\right),
\end{aligned}
$$

with $\mathbf{q}_{k}$ (or $\mathbf{y}_{k}$ ) and $\mathbf{d}_{i}$ denoting the $k$-th and $i$-th column of $\mathbf{Q}$ (or $\mathbf{Y})$ and $\mathbf{D}$, respectively, while $\operatorname{diag}\left(\mathbf{D}^{T} \mathbf{D}\right)$ denotes a diagonal matrix with elements on the main diagonal identical to those of the matrix $\mathbf{D}^{T} \mathbf{D}$. Note that in the approximate function w.r.t. $\mathbf{P}$ and $\mathbf{Q}$, the remaining variables $(\mathbf{Q}, \mathbf{S})$ and $(\mathbf{P}, \mathbf{S})$ are fixed, respectively. Although it is tempting to define the approximate function of $f(\mathbf{P}, \mathbf{Q}, \mathbf{S})$ w.r.t. $\mathbf{S}$ by fixing $\mathbf{P}$ and $\mathbf{Q}$, minimizing $f\left(\mathbf{P}^{t}, \mathbf{Q}^{t}, \mathbf{S}\right)$ w.r.t. the matrix variable $\mathbf{S}$ does not have a closed-form solution and must be solved iteratively. Therefore the proposed approximate function $\tilde{f}_{S}\left(\mathbf{S} ; \mathbf{Z}^{t}\right)$ in $(31 \mathrm{c})$ consists of $I K$ component functions, and in the $(i, k)$-th component function, $s_{i, k}$ is the variable while all other variables are fixed, namely, $\mathbf{P}, \mathbf{Q},\left(s_{j, k}\right)_{j \neq i}$, and $\left(\mathbf{s}_{j}\right)_{j \neq i}$. As we will show shortly, minimizing $\tilde{f}\left(\mathbf{S} ; \mathbf{Z}^{t}\right)$ w.r.t. $\mathbf{S}$ exhibits a closed-form solution.

We remark that the approximate function $\tilde{f}\left(\mathbf{Z} ; \mathbf{Z}^{t}\right)$ is a (strongly) convex function and it is differentiable in both $\mathbf{Z}$ and $\mathbf{Z}^{t}$. Furthermore, the gradient of the approximate function $\tilde{f}\left(\mathbf{P}, \mathbf{Q}, \mathbf{S} ; \mathbf{Z}^{t}\right)$ is equal to that of $f(\mathbf{P}, \mathbf{Q}, \mathbf{S})$ at $\mathbf{Z}=\mathbf{Z}^{t}$. To see this:

$$
\nabla_{\mathbf{P}} \tilde{f}\left(\mathbf{Z} ; \mathbf{Z}^{t}\right)=\nabla_{\mathbf{P}} \tilde{f}_{P}\left(\mathbf{P} ; \mathbf{Z}^{t}\right)=\left.\nabla_{\mathbf{P}} f\left(\mathbf{P}, \mathbf{Q}^{t}, \mathbf{S}^{t}\right)\right|_{\mathbf{P}=\mathbf{P}^{t}},
$$

and similarly $\nabla_{\mathbf{Q}} \tilde{f}\left(\mathbf{Z} ; \mathbf{Z}^{t}\right)=\left.\nabla_{\mathbf{Q}} f(\mathbf{P}, \mathbf{Q}, \mathbf{S})\right|_{\mathbf{Z}=\mathbf{Z}^{t}}$. Furthermore, $\nabla_{\mathbf{S}} \tilde{f}\left(\mathbf{Z} ; \mathbf{Z}^{t}\right)=\left(\nabla_{s_{i, k}} \tilde{f}\left(\mathbf{Z} ; \mathbf{Z}^{t}\right)\right)_{i, k}$ while

$$
\begin{aligned}
\nabla_{s_{i, k}} \tilde{f}\left(\mathbf{Z} ; \mathbf{Z}^{t}\right) & =\nabla_{s_{i, k}} \tilde{f}_{S}\left(\mathbf{S} ; \mathbf{Z}^{t}\right) \\
& =\nabla_{s_{i, k}} f\left(\mathbf{P}^{t}, \mathbf{Q}^{t}, s_{i, k}, \mathbf{s}_{i,-k}^{t}, \mathbf{s}_{-i}^{t}\right) \\
& =\left.\nabla_{s_{i, k}} f(\mathbf{P}, \mathbf{Q}, \mathbf{S})\right|_{\mathbf{Z}=\mathbf{Z}^{t}} .
\end{aligned}
$$

Therefore Assumptions (A1)-(A3) are satisfied.

In iteration $t$, the approximate problem consists of minimizing the approximate function:

$$
\underset{\mathbf{Z}=(\mathbf{P}, \mathbf{Q}, \mathbf{S})}{\operatorname{minimize}} \underbrace{\tilde{f}_{P}\left(\mathbf{P} ; \mathbf{Z}^{t}\right)+\tilde{f}_{Q}\left(\mathbf{Q} ; \mathbf{Z}^{t}\right)+\tilde{f}_{S}\left(\mathbf{S} ; \mathbf{Z}^{t}\right)}_{\tilde{f}\left(\mathbf{Z} ; \mathbf{Z}^{t}\right)}+g(\mathbf{S}) .
$$

Since $\tilde{f}\left(\mathbf{Z} ; \mathbf{Z}^{t}\right)$ is strongly convex in $\mathbf{Z}$ and $g(\mathbf{S})$ is a convex function w.r.t. $\mathbf{S}$, the approximate problem (32) is strongly convex and it has a unique globally optimal solution, which is denoted as $\mathbb{B} \mathbf{Z}^{t}=\left(\mathbb{B}_{P} \mathbf{Z}^{t}, \mathbb{B}_{Q} \mathbf{Z}^{t}, \mathbb{B}_{S} \mathbf{Z}^{t}\right)$. As the approximate problem (32) is separable among the optimization variables $\mathbf{P}, \mathbf{Q}$ and $\mathbf{S}$, it naturally decomposes into several smaller problems which can be solved in parallel:

$$
\begin{aligned}
\mathbb{B}_{P} \mathbf{Z}^{t} & \triangleq \underset{\mathbf{P}_{k}}{\arg \min } \tilde{f}_{P}\left(\mathbf{P} ; \mathbf{Z}^{t}\right) \\
& =\left(\mathbf{Y}-\mathbf{D S}^{t}\right)\left(\mathbf{Q}^{t}\right)^{T}\left(\mathbf{Q}^{t}\left(\mathbf{Q}^{t}\right)^{T}+\lambda \mathbf{I}\right)^{-1}, \\
\mathbb{B}_{Q} \mathbf{Z}^{t} & \triangleq \underset{\mathbf{Q}}{\arg \min } \tilde{f}_{Q}\left(\mathbf{Q} ; \mathbf{Z}^{t}\right) \\
& =\left(\left(\mathbf{P}^{t}\right)^{T} \mathbf{P}^{t}+\lambda \mathbf{I}\right)^{-1}\left(\mathbf{P}^{t}\right)^{T}\left(\mathbf{Y}-\mathbf{D S}^{t}\right),
\end{aligned}
$$




$$
\begin{aligned}
\mathbb{B}_{S} \mathbf{Z}^{t} \triangleq & \underset{\mathbf{S}}{\arg \min } \tilde{f}_{S}\left(\mathbf{S} ; \mathbf{Z}^{t}\right)+g(\mathbf{S}) \\
= & \operatorname{diag}\left(\mathbf{D}^{T} \mathbf{D}\right)^{-1} . \\
& \mathcal{S}_{\mu}\left(\operatorname{diag}\left(\mathbf{D}^{T} \mathbf{D}\right) \mathbf{S}^{t}-\mathbf{D}^{T}\left(\mathbf{D} \mathbf{S}^{t}-\mathbf{Y}^{t}+\mathbf{P}^{t} \mathbf{Q}^{t}\right)\right),
\end{aligned}
$$

where $\mathcal{S}_{\mu}(\mathbf{X})$ is an element-wise soft-thresholding operator: the $(i, j)$-th element of $\mathcal{S}_{\mu}(\mathbf{X})$ is $\left[X_{i j}-\lambda\right]^{+}-\left[-X_{i j}-\lambda\right]^{+}$. As we can readily see from (33), the approximate problems can be solved efficiently because the optimal solutions are provided in an analytical expression.

Since $\tilde{f}\left(\mathbf{Z} ; \mathbf{Z}^{t}\right)$ is convex in $\mathbf{Z}$ and differentiable in both $\mathbf{Z}$ and $\mathbf{Z}^{t}$, and has the same gradient as $f(\mathbf{Z})$ at $\mathbf{Z}=\mathbf{Z}^{t}$, it follows from Proposition 1 that $\mathbb{B} \mathbf{Z}^{t}-\mathbf{Z}^{t}$ is a descent direction of the original objective function $f(\mathbf{Z})+g(\mathbf{S})$ at $\mathbf{Z}=\mathbf{Z}^{t}$. The variable update in the $t$-th iteration is thus defined as follows:

$$
\begin{aligned}
\mathbf{P}^{t+1} & =\mathbf{P}^{t}+\gamma\left(\mathbb{B}_{P} \mathbf{Z}^{t}-\mathbf{P}^{t}\right), \\
\mathbf{Q}^{t+1} & =\mathbf{Q}^{t}+\gamma\left(\mathbb{B}_{Q} \mathbf{Z}^{t}-\mathbf{Q}^{t}\right), \\
\mathbf{S}^{t+1} & =\mathbf{S}^{t}+\gamma\left(\mathbb{B}_{S} \mathbf{Z}^{t}-\mathbf{S}^{t}\right),
\end{aligned}
$$

where $\gamma \in(0,1]$ is the stepsize that should be properly selected.

We determine the stepsize $\gamma$ by the proposed exact line search scheme (21):

$$
f\left(\mathbf{Z}^{t}+\gamma\left(\mathbb{B} \mathbf{Z}^{t}-\mathbf{Z}^{t}\right)\right)+g\left(\mathbf{S}^{t}\right)+\gamma\left(g\left(\mathbb{B}_{S} \mathbf{Z}^{t}\right)-g\left(\mathbf{S}^{t}\right)\right) .
$$

After substituting the expressions of $f(\mathbf{Z})$ and $g(\mathbf{S})$ into (35), the exact line search consists in minimizing a fourth order polynomial over the interval $[0,1]$ :

$$
\begin{aligned}
\gamma^{t} & =\underset{0 \leq \gamma \leq 1}{\arg \min }\left\{f\left(\mathbf{Z}^{t}+\gamma\left(\mathbb{B} \mathbf{Z}^{t}-\mathbf{Z}^{t}\right)\right)+\gamma\left(g\left(\mathbb{B}_{S} \mathbf{X}^{t}\right)-g\left(\mathbf{S}^{t}\right)\right)\right\} \\
& =\underset{0 \leq \gamma \leq 1}{\arg \min }\left\{\frac{1}{4} a \gamma^{4}+\frac{1}{3} b \gamma^{3}+\frac{1}{2} c \gamma^{2}+d \gamma\right\},
\end{aligned}
$$

where

$$
\begin{aligned}
a \triangleq & 2\left\|\triangle \mathbf{P}^{t} \triangle \mathbf{Q}^{t}\right\|_{F}^{2}, \\
b \triangleq & 3 \operatorname{tr}\left(\triangle \mathbf{P}^{t} \triangle \mathbf{Q}^{t}\left(\mathbf{P}^{t} \triangle \mathbf{Q}^{t}+\triangle \mathbf{P}^{t} \mathbf{Q}^{t}+\mathbf{D} \triangle \mathbf{S}^{t}\right)^{T}\right), \\
c \triangleq & 2 \operatorname{tr}\left(\triangle \mathbf{P}^{t} \triangle \mathbf{Q}^{t}\left(\mathbf{P}^{t} \mathbf{Q}^{t}+\mathbf{D} \mathbf{S}^{t}-\mathbf{Y}^{t}\right)^{T}\right) \\
& +\left\|\mathbf{P}^{t} \triangle \mathbf{Q}^{t}+\triangle \mathbf{P}^{t} \mathbf{Q}^{t}+\mathbf{D} \triangle \mathbf{S}^{t}\right\|_{F}^{2} \\
& +\lambda\left(\left\|\triangle \mathbf{P}^{t}\right\|_{F}^{2}+\left\|\triangle \mathbf{Q}^{t}\right\|_{F}^{2}\right), \\
d \triangleq & \operatorname{tr}\left(\left(\mathbf{P}^{t} \triangle \mathbf{Q}^{t}+\triangle \mathbf{P}^{t} \mathbf{Q}^{t}+\mathbf{D} \triangle \mathbf{S}^{t}\right)\left(\mathbf{P}^{t} \mathbf{Q}^{t}+\mathbf{D} \mathbf{S}^{t}-\mathbf{Y}^{t}\right)\right) \\
& +\lambda\left(\operatorname{tr}\left(\mathbf{P}^{t} \triangle \mathbf{P}^{t}\right)+\operatorname{tr}\left(\mathbf{Q}^{t} \triangle \mathbf{Q}^{t}\right)\right)+\mu\left(\left\|\mathbb{B}_{S} \mathbf{X}^{t}\right\|_{1}-\left\|\mathbf{S}^{t}\right\|_{1}\right),
\end{aligned}
$$

for $\triangle \mathbf{P}^{t} \triangleq \mathbb{B}_{P} \mathbf{Z}^{t}-\mathbf{P}^{t}, \triangle \mathbf{Q}^{t} \triangleq \mathbb{B}_{Q} \mathbf{Z}^{t}-\mathbf{Q}^{t}$ and $\triangle \mathbf{S}^{t} \triangleq \mathbb{B}_{S} \mathbf{Z}^{t}-$ $\mathbf{S}^{t}$. Finding the optimal points of (36) is equivalent to finding the nonnegative real root of a third-order polynomial. Making use of Cardano's method, we write $\gamma^{t}$ defined in (36) as the closed-form expression:

$$
\begin{aligned}
& \gamma^{t}=\left[\bar{\gamma}^{t}\right]_{0}^{1}, \\
& \bar{\gamma}^{t}=\sqrt[3]{\Sigma_{1}+\sqrt{\Sigma_{1}^{2}+\Sigma_{2}^{3}}}+\sqrt[3]{\Sigma_{1}-\sqrt{\Sigma_{1}^{2}+\Sigma_{2}^{3}}}-\frac{b}{3 a},
\end{aligned}
$$

where $[x]_{0}^{1}=\max (\min (x, 1), 0)$ is the projection of $x$ onto the interval $[0,1], \Sigma_{1} \triangleq-(b / 3 a)^{3}+b c / 6 a^{2}-d / 2 a$ and $\Sigma_{2} \triangleq c / 3 a-$ $(b / 3 a)^{2}$. Note that in (37b), the right hand side contains three values (two of them can attain complex numbers), and the equal sign must be interpreted as assigning the smallest real nonnegative values.

The proposed algorithm is summarized in Algorithm 2, which we name as the Soft-Thresholding with Exact Line search Algorithm
Algorithm 2 STELA: The proposed parallel BCD algorithm with exact line search for the sparsity regularized rank minimization problem (29)

Data: $t=0, \mathbf{Z}^{0}$ (arbitrary but fixed), stop criterion $\delta$.

S1: Compute $\left(\mathbb{B}_{P} \mathbf{Z}^{t}, \mathbb{B}_{Q} \mathbf{Z}^{t}, \mathbb{B}_{S} \mathbf{Z}^{t}\right)$ according to (33).

S2: Determine the stepsize $\gamma^{t}$ by the exact line search (37).

S3: Update $(\mathbf{P}, \mathbf{Q}, \mathbf{Z})$ according to (34).

S4: If $\left|\operatorname{tr}\left(\left(\mathbb{B} \mathbf{Z}^{t}-\mathbf{Z}^{t}\right)^{T} \nabla f\left(\mathbf{Z}^{t}\right)\right)+g\left(\mathbb{B}_{S} \mathbf{Z}^{t}\right)-g\left(\mathbf{S}^{t}\right)\right| \leq \delta$, STOP; otherwise $t \leftarrow t+1$ and go to $\mathbf{S 1}$.

(STELA). We draw a few comments on its attractive features and compare it with state-of-the-art algorithms proposed for problem (29).

i) Fast convergence. In each iteration, the variables $\mathbf{P}, \mathbf{Q}$, and $\mathbf{S}$ are updated simultaneously based on the best-response. The improvement in convergence speed w.r.t. the $\mathrm{BCD}$ algorithm in [12] is notable because in the BCD algorithm, the optimization w.r.t. each row of $\mathbf{S}$ is implemented in a sequential order, and the number of rows is usually very large in big data applications. To avoid the meticulous choice of stepsizes and further accelerate the convergence, the stepsize is calculated by the exact line search and it yields faster convergence than SCA algorithms with diminishing stepsizes $[15,16]$.

ii) Low complexity. The proposed algorithm STELA has a very low complexity, because both the best-responses $\left(\mathbb{B}_{P} \mathbf{Z}^{t}, \mathbb{B}_{Q} \mathbf{Z}^{t}, \mathbb{B}_{S} \mathbf{Z}^{t}\right)$ and the exact line search can be computed by closed-form expressions, cf. (31) and (37). Note that computing $\mathbb{B}_{P} \mathbf{Z}^{t}$ and $\mathbb{B}_{Q} \mathbf{Z}^{t}$ according to (33a)-(33b) involves a matrix inverse. This is usually affordable because the matrices to be inverted are of a dimension $\rho \times \rho$ while $\rho$ is usually small. Furthermore, the matrix inverse operation could be saved by adopting an element-wise decomposition for $\mathbf{P}$ and $\mathbf{Q}$ that is in the same essence as $\mathbf{S}$ in (31c).

iii) Guaranteed convergence. Since $f(\mathbf{P}, \mathbf{Q}, \mathbf{S})$ is nonconvex and $\nabla f(\mathbf{P}, \mathbf{Q}, \mathbf{S})$ is not Lipschitz continuous, the ADMM algorithm [19] and SCA algorithms with diminishing stepsizes [15, 16] do not necessarily converge. By comparison, the proposed algorithm STELA has a guaranteed convergence in the sense that every limit point of the sequence $\left\{\mathbf{Z}^{t}\right\}_{t}$ is a stationary point of problem (29).

Parallel Decomposition and Implementation of the Proposed Algorithm STELA

The proposed algorithm STELA can be further decomposed to enable the parallel processing over a number of $L$ nodes in a distributed network. To see this, we first decompose the system model across the nodes:

$$
\mathbf{Y}_{l}=\mathbf{X}_{l}+\mathbf{D}_{l} \mathbf{S}+\mathbf{V}_{l}, l=1, \ldots, L
$$

where $\mathbf{Y}_{l} \in \mathbb{R}^{N_{l} \times K}, \mathbf{X}_{l} \in \mathbb{R}^{N_{l} \times K}, \mathbf{D}_{l} \in \mathbb{R}^{N_{l} \times I}$ and $\mathbf{V}_{l} \in \mathbb{R}^{N_{l} \times K}$ consists of $N_{l}$ rows of $\mathbf{Y}, \mathbf{X}, \mathbf{D}$ and $\mathbf{V}$, respectively:

$\mathbf{Y}=\left[\begin{array}{c}\mathbf{Y}_{1} \\ \mathbf{Y}_{2} \\ \vdots \\ \mathbf{Y}_{L}\end{array}\right], \mathbf{X}=\left[\begin{array}{c}\mathbf{X}_{1} \\ \mathbf{X}_{2} \\ \vdots \\ \mathbf{X}_{L}\end{array}\right], \mathbf{D}=\left[\begin{array}{c}\mathbf{D}_{1} \\ \mathbf{D}_{2} \\ \vdots \\ \mathbf{D}_{L}\end{array}\right], \mathbf{V}=\left[\begin{array}{c}\mathbf{V}_{1} \\ \mathbf{V}_{2} \\ \vdots \\ \mathbf{V}_{L}\end{array}\right]$ 
Since the variables of interest for the node $l$ are $\mathbf{X}_{k}$ and $\mathbf{S}$, we decompose $\mathbf{P}$ into multiple blocks $\left(\mathbf{P}_{l}\right)_{l=1}^{L}$ with $\mathbf{P}_{l} \in \mathbb{R}^{N_{l} \times \rho}$ :

$$
\mathbf{P}=\left[\begin{array}{c}
\mathbf{P}_{1} \\
\mathbf{P}_{2} \\
\vdots \\
\mathbf{P}_{L}
\end{array}\right]
$$

All nodes should have access to the variable $\mathbf{Q}$ so that $\mathbf{X}_{l}$ can be estimated locally by $\mathbf{X}_{l}=\mathbf{P}_{l} \mathbf{Q}$.

The computation of $\mathbb{B}_{P} \mathbf{Z}^{t}$ in (34a) can be decomposed as $\mathbb{B}_{P} \mathbf{Z}^{t}=$ $\left(\mathbb{B}_{P, l} \mathbf{Z}^{t}\right)_{l=1}^{L}$ :

$$
\mathbb{B}_{P, l} \mathbf{Z}^{t}=\left(\mathbf{Y}_{l}-\mathbf{D}_{l} \mathbf{S}^{t}\right)\left(\mathbf{Q}^{t}\right)^{T}\left(\mathbf{Q}^{t}\left(\mathbf{Q}^{t}\right)^{T}+\lambda \mathbf{I}\right)^{-1}, l=1, \ldots, L .
$$

Accordingly, the computation of $\mathbb{B}_{Q} \mathbf{Z}^{t}$ and $\mathbb{B}_{S} \mathbf{Z}^{t}$ in (34b) and (34c) can be rewritten as

$$
\begin{aligned}
& \mathbb{B}_{Q} \mathbf{Z}^{t}=\left(\sum_{l=1}^{L}\left(\mathbf{P}_{l}^{t}\right)^{T} \mathbf{P}_{l}^{t}+\lambda \mathbf{I}\right)^{-1}\left(\sum_{l=1}^{L}\left(\mathbf{P}_{l}^{t}\right)^{T}\left(\mathbf{Y}_{l}-\mathbf{D}_{l} \mathbf{S}^{t}\right)\right), \\
& \mathbb{B}_{S} \mathbf{Z}^{t}=\operatorname{diag}\left(\sum_{l=1}^{L} \mathbf{D}_{l}^{T} \mathbf{D}_{l}\right)^{-1} . \\
& \mathcal{S}_{\mu}\left(\operatorname{diag}\left(\sum_{l=1}^{L} \mathbf{D}_{l}^{T} \mathbf{D}_{l}\right) \mathbf{S}^{t}-\sum_{l=1}^{L} \mathbf{D}_{l}^{T}\left(\mathbf{D}_{l} \mathbf{S}^{t}-\mathbf{Y}_{l}^{t}+\mathbf{P}_{l}^{t} \mathbf{Q}^{t}\right)\right) .
\end{aligned}
$$

Before determining the stepsize, the computation of $a$ in (37) can also be decomposed among the nodes as $a=\sum_{l=1}^{L} a_{l}$, where

$$
a_{l} \triangleq 2\left\|\triangle \mathbf{P}_{l}^{t} \triangle \mathbf{Q}^{t}\right\|_{F}^{2} .
$$

The decomposition of $b, c$, and $d$ is similar to that of $a$, where

$$
\begin{aligned}
b_{l} \triangleq & 3 \operatorname{tr}\left(\triangle \mathbf{P}_{l}^{t} \triangle \mathbf{Q}^{t}\left(\mathbf{P}_{l}^{t} \triangle \mathbf{Q}^{t}+\triangle \mathbf{P}_{l}^{t} \mathbf{Q}^{t}+\mathbf{D}_{l} \triangle \mathbf{S}^{t}\right)^{T}\right), \\
c_{l} \triangleq & 2 \operatorname{tr}\left(\triangle \mathbf{P}_{l}^{t} \triangle \mathbf{Q}^{t}\left(\mathbf{P}_{l}^{t} \mathbf{Q}^{t}+\mathbf{D}_{l} \mathbf{S}^{t}-\mathbf{Y}_{l}^{t}\right)^{T}\right) \\
& +\left\|\mathbf{P}_{l}^{t} \triangle \mathbf{Q}^{t}+\triangle \mathbf{P}_{l}^{t} \mathbf{Q}^{t}+\mathbf{D}_{l} \triangle \mathbf{S}^{t}\right\|_{F}^{2} \\
& +\lambda\left\|\triangle \mathbf{P}_{l}^{t}\right\|_{F}^{2}+\frac{\lambda}{I}\left\|\triangle \mathbf{Q}_{l}^{t}\right\|_{F}^{2}, \\
d_{l} \triangleq & \operatorname{tr}\left(\left(\mathbf{P}_{l}^{t} \triangle \mathbf{Q}^{t}+\triangle \mathbf{P}_{l}^{t} \mathbf{Q}^{t}+\mathbf{D}_{l} \triangle \mathbf{S}^{t}\right)\left(\mathbf{P}_{l}^{t} \mathbf{Q}^{t}+\mathbf{D}_{l} \mathbf{S}^{t}-\mathbf{Y}_{l}^{t}\right)\right) \\
& +\lambda \operatorname{tr}\left(\mathbf{P}_{l}^{t} \triangle \mathbf{P}_{l}^{t}\right)+\frac{\lambda}{I} \operatorname{tr}\left(\mathbf{Q}^{t} \triangle \mathbf{Q}^{t}\right)+\frac{\mu}{I}\left(\left\|\mathbb{B}_{S} \mathbf{X}^{t}\right\|_{1}-\left\|\mathbf{S}^{t}\right\|_{1}\right) .
\end{aligned}
$$

To compute the stepsize as in (37), the nodes mutually exchange $\left(a_{l}, b_{l}, c_{l}, d_{l}\right)$. The four dimensional vector $\left(a_{l}, b_{l}, c_{l}, d_{l}\right)$ provides each node with all the necessary information to individually calculate $(a, b, c, d)$ and $\left(\Sigma_{1}, \Sigma_{2}, \Sigma_{3}\right)$, and then the stepsize $\gamma^{t}$ according to (37). The signaling incurred by the exact line search is thus small and affordable.

\section{Numerical Simulations}

We perform numerical tests to compare the proposed algorithm STELA with the BCD algorithm [12] and the ADMM algorithm [19]. We start with a brief description of the ADMM algorithm: the problem (29) can be rewritten as

$\underset{\mathbf{P}, \mathbf{Q}, \mathbf{A}, \mathbf{B}}{\operatorname{minimize}} \frac{1}{2}\|\mathbf{P Q}+\mathbf{D A}-\mathbf{Y}\|_{F}^{2}+\frac{\lambda}{2}\left(\|\mathbf{P}\|_{F}^{2}+\|\mathbf{Q}\|_{F}^{2}\right)+\mu\|\mathbf{B}\|_{1}$ subject to $\mathbf{A}=\mathbf{B}$.

The augmented Lagrangian of (38) is

$$
\begin{aligned}
L_{c}(\mathbf{P}, \mathbf{Q}, \mathbf{A}, \mathbf{B}, \boldsymbol{\Pi})= & \frac{1}{2}\|\mathbf{P Q}+\mathbf{D A}-\mathbf{Y}\|_{F}^{2}+\frac{\lambda}{2}\left(\|\mathbf{P}\|_{F}^{2}+\|\mathbf{Q}\|_{F}^{2}\right) \\
& +\mu\|\mathbf{B}\|_{1}+\operatorname{tr}\left(\mathbf{\Pi}^{T}(\mathbf{A}-\mathbf{B})\right)+\frac{c}{2}\|\mathbf{A}-\mathbf{B}\|_{F}^{2},
\end{aligned}
$$

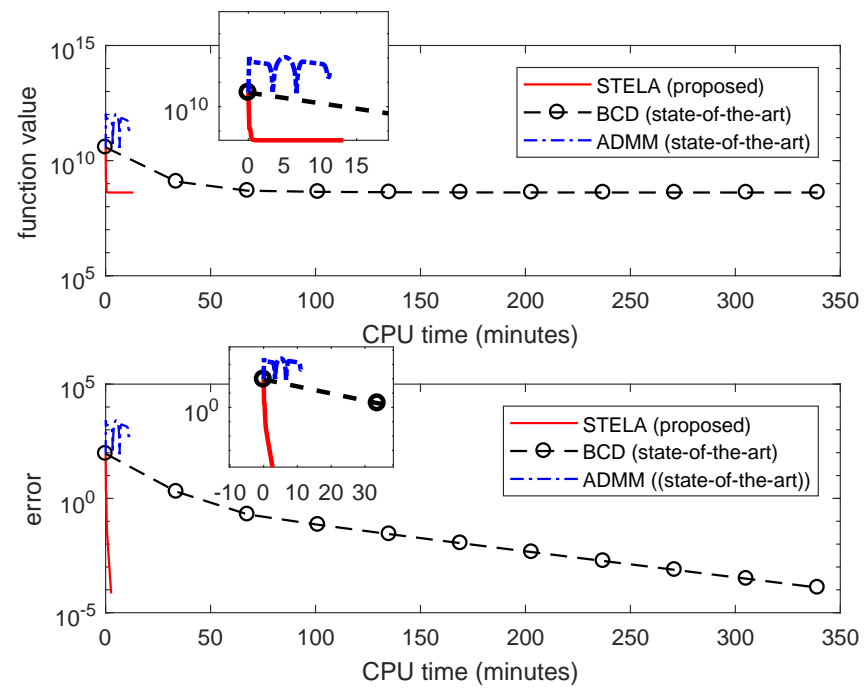

Figure 2. Sparsity regularized rank minimization: achieved function value $h\left(\mathbf{Z}^{t}\right)$ versus the number of iterations and CPU time (in minutes).

where $c$ is a positive constant. In ADMM, the variables are updated in the $t$-th iteration as follows:

$$
\begin{aligned}
\left(\mathbf{Q}^{t+1}, \mathbf{B}^{t+1}\right) & =\underset{\mathbf{Q}, \mathbf{A}}{\arg \min } L_{c}\left(\mathbf{P}^{t}, \mathbf{Q}, \mathbf{A}^{t}, \mathbf{B}, \mathbf{\Pi}^{t}\right), \\
\mathbf{P}^{t+1} & =\underset{\mathbf{P}}{\arg \min } L_{c}\left(\mathbf{P}, \mathbf{Q}^{t+1}, \mathbf{A}^{t+1}, \mathbf{B}^{t}, \mathbf{\Pi}^{t}\right), \\
\mathbf{A}^{t+1} & =\underset{\mathbf{B}}{\arg \min } L_{c}\left(\mathbf{P}^{t+1}, \mathbf{Q}^{t+1}, \mathbf{A}, \mathbf{B}^{t+1}, \mathbf{\Pi}^{t}\right), \\
\mathbf{\Pi}^{t+1} & =\mathbf{\Pi}^{t}+c\left(\mathbf{A}^{t+1}-\mathbf{B}^{t+1}\right) .
\end{aligned}
$$

Note that the solutions to the above optimization problems have an analytical expression [19]. We set $c=10^{4}$.

The simulation parameters are set as follows. $N=1000, K=$ $4000, I=4000, \rho=10$. The elements of $\mathbf{D}$ are binary and generated randomly and they are either 0 or 1 . The elements of $\mathbf{V}$ follow the Gaussian distribution with mean 0 and variance 0.01 . Each element of $\mathbf{S}$ can take three possible values, namely, $-1,0,1$, with the probability $P\left(S_{i, k}=-1\right)=P\left(S_{i k}=1\right)=0.05$ and $P\left(S_{i k}=0\right)=0.9$. We set $\mathbf{Y}=\mathbf{P Q}+\mathbf{D S}+\mathbf{V}$, where $\mathbf{P}$ and $\mathbf{Q}$ are generated randomly following the Gaussian distribution $\mathcal{N}(\mathbf{0}, 100 / I)$ and $\mathcal{N}(\mathbf{0}, 100 / K)$, respectively. The sparsity regularization parameters are $\lambda=0.1 \cdot\|\mathbf{Y}\|$ $(\|\mathbf{Y}\|$ is the spectral norm of $\mathbf{Y})$ and $\mu=0.1 \cdot\left\|\mathbf{D}^{T} \mathbf{Y}\right\|_{\infty}$. The simulation results are averaged over 20 realizations. For the visual convenience, the curves of STELA and ADMM are magnified in a small window inside the same figure.

In Fig. 2 (a) and (b), we show respectively the achieved objective function value and error versus the CPU time (in minutes) by different algorithms, namely, STELA, BCD and ADMM. In Fig. 2 (b), the error is defined as $\left(f\left(\mathbf{Z}^{t}\right)+g\left(\mathbf{S}^{t}\right)-f\left(\mathbf{Z}^{\star}\right)-g\left(\mathbf{S}^{\star}\right)\right) /\left(f\left(\mathbf{Z}^{\star}\right)+g\left(\mathbf{S}^{\star}\right)\right)$, where $\mathbf{Z}^{\star}$ is obtained by running the proposed algorithm STELA for a sufficiently large number of iterations. As we see from Fig. 2 (a), the ADMM does not converge, as the optimization problem (38) (and (29)) is nonconvex. We also observe that the behavior of the ADMM is very sensitive to the value of $c$ : in some instances, the ADMM may converge if $c$ is large enough, but it is a difficult task on its own to choose an appropriate value of $c$ to achieve a good performance.

We run the BCD algorithm for 10 iterations, each represented by a circle. In each iteration, all rows of $\mathbf{S}$ are updated once in a sequential order, and it incurs a large delay. In particular, we see from Fig. 2 (a) that each iteration of the BCD algorithm takes about 35 minutes, and a reasonably good solution is obtained after two iterations (70 
minutes). By contrast, all variables are updated simultaneously in STELA and the CPU time needed for each iteration is very small. We see from Fig. 2 (b) that STELA converges to a stationary point with a precision of $10^{-5}$ in less than 1 minute, while it takes the BCD algorithm about 330 minutes (5.5 hours) to find a solution that has the same precision. This marks a notable improvement which is important in real time anomaly detection in large networks.

\section{B. Network anomaly detection based on nonconvex regularization} functions

In this subsection, we apply the proposed SCA algorithm to find a stationary point of (6), which we duplicate here for the simplicity of cross-reference:

$$
\begin{aligned}
\underset{\mathbf{X}, \mathbf{S}}{\operatorname{minimize}} & \underbrace{\frac{1}{2}\|\mathbf{X}+\mathbf{D S}-\mathbf{Y}\|_{F}^{2}}_{f(\mathbf{X}, \mathbf{S})}+\underbrace{\lambda \kappa_{0}\|\mathbf{X}\|_{*}+\mu\|\mathbf{S}\|_{1}}_{g^{+}(\mathbf{X}, \mathbf{S})} \\
- & \underbrace{\left.\lambda \kappa_{0} \sum_{n=1}^{N} \sigma_{n}(\mathbf{X})-\lambda \sum_{n=1}^{N} \kappa\left(\sigma_{n}(\mathbf{X})\right)\right)}_{g^{-}(\mathbf{X})},
\end{aligned}
$$

where $g^{-}(\mathbf{X})$ is convex and differentiable [33, Prop. 5].

Since $f(\mathbf{X}, \mathbf{S})$ is convex in $(\mathbf{X}, \mathbf{S})$, we adopt the best-response type approximation at a given point $\mathbf{Z}^{t}=\left(\mathbf{X}^{t}, \mathbf{S}^{t}\right)$, namely,

$$
\tilde{f}\left(\mathbf{X}, \mathbf{S} ; \mathbf{Z}^{t}\right)=\tilde{f}_{X}\left(\mathbf{X} ; \mathbf{Z}^{t}\right)+\tilde{f}_{S}\left(\mathbf{S} ; \mathbf{Z}^{t}\right),
$$

where

$$
\begin{aligned}
\tilde{f}_{X}\left(\mathbf{X} ; \mathbf{Z}^{t}\right) & \triangleq f\left(\mathbf{X}, \mathbf{S}^{t}\right)=\frac{1}{2}\left\|\mathbf{X}+\mathbf{D S}^{t}-\mathbf{Y}\right\|_{F}^{2} \\
\tilde{f}_{S}\left(\mathbf{S} ; \mathbf{Z}^{t}\right) & \triangleq \sum_{i, k} f\left(\mathbf{P}^{t}, \mathbf{Q}^{t}, s_{i, k},\left(s_{j, k}^{t}\right)_{j \neq i},\left(\mathbf{s}_{j}^{t}\right)_{j \neq i}\right) \\
& =\sum_{i, k} \frac{1}{2}\left\|\mathbf{P}^{t} \mathbf{q}_{k}^{t}+\mathbf{d}_{i} s_{i, k}+\sum_{j \neq i} \mathbf{d}_{j} s_{j, k}^{t}-\mathbf{y}_{k}\right\|_{2}^{2} .
\end{aligned}
$$

In iteration $t$, the approximate problem consists of minimizing the approximate function:

$\underset{\mathbf{X}, \mathbf{S}}{\operatorname{minimize}}\left\{\begin{array}{l}\tilde{f}_{X}\left(\mathbf{X} ; \mathbf{Z}^{t}\right)+\tilde{f}_{S}\left(\mathbf{S} ; \mathbf{Z}^{t}\right)-\left\langle\mathbf{X}-\mathbf{X}^{t}, \nabla g^{-}\left(\mathbf{X}^{t}\right)\right\rangle \\ +g^{+}(\mathbf{X}, \mathbf{S})\end{array}\right\}$,

where $\langle\mathbf{A}, \mathbf{B}\rangle \triangleq \operatorname{tr}\left(\mathbf{A}^{T} \mathbf{B}\right)$. Its optimal point, denoted as $\mathbb{B} \mathbf{Z}^{t}=$ $\left(\mathbb{B}_{X} \mathbf{Z}^{t}, \mathbb{B}_{S} \mathbf{Z}^{t}\right)$, has a closed-form expression:

$$
\begin{aligned}
\mathbb{B}_{X} \mathbf{Z}^{t} & =\underset{\mathbf{X}}{\arg \max } \tilde{f}_{X}\left(\mathbf{X} ; \mathbf{Z}^{t}\right)-\left\langle\mathbf{X}-\mathbf{X}^{t}, \nabla g^{-}\left(\mathbf{X}^{t}\right)\right\rangle+\lambda \kappa_{0}\|\mathbf{X}\|_{*} \\
& =\mathbf{U}^{t} \max \left(\mathbf{D}^{t}-\lambda \kappa_{0} \mathbf{I}, \mathbf{0}\right)\left(\mathbf{V}^{t}\right)^{T}, \\
\mathbb{B}_{S} \mathbf{Z}^{t} & =\underset{\mathbf{S}}{\arg \max } \tilde{f}_{S}\left(\mathbf{S} ; \mathbf{Z}^{t}\right)+\mu\|\mathbf{S}\|_{1} \\
& =\operatorname{diag}\left(\mathbf{D}^{T} \mathbf{D}\right)^{-1} \mathcal{S}_{\mu}\left(\operatorname{diag}\left(\mathbf{D}^{T} \mathbf{D}\right) \mathbf{S}^{t}-\mathbf{D}^{T}\left(\mathbf{D} \mathbf{S}^{t}-\mathbf{Y}^{t}+\mathbf{X}^{t}\right)\right),
\end{aligned}
$$

where $\mathbf{Y}-\mathbf{D} \mathbf{S}^{t}+\nabla g^{-}\left(\mathbf{X}^{t}\right)=\mathbf{U}^{t} \mathbf{D}^{t}\left(\mathbf{V}^{t}\right)^{T}$ is the singular value decomposition (SVD) of $\mathbf{Y}-\mathbf{D S}^{t}+\nabla g^{-}\left(\mathbf{X}^{t}\right)$.

We determine the stepsize by the proposed exact line search (21):

$$
\underset{0 \leq \gamma \leq 1}{\operatorname{minimize}}\left\{\begin{array}{l}
f\left(\mathbf{X}^{t}+\gamma\left(\mathbb{B}_{X} \mathbf{Z}^{t}-\mathbf{X}^{t}\right), \mathbf{X}^{t}+\gamma\left(\mathbb{B}_{S} \mathbf{Z}^{t}-\mathbf{S}^{t}\right)\right) \\
-\gamma\left\langle\mathbb{B}_{X} \mathbf{Z}^{t}-\mathbf{X}^{t}, \nabla g^{-}\left(\mathbf{X}^{t}\right)\right\rangle \\
+\gamma\left(g^{+}\left(\mathbb{B}_{X} \mathbf{Z}^{t}, \mathbb{B}_{S} \mathbf{Z}^{t}\right)-g^{+}\left(\mathbf{X}^{t}, \mathbf{S}^{t}\right)\right)
\end{array}\right\} .
$$

This is a convex quadratic problem and its solution has a simple closed-form expression; we leave the details to the reader.
After the stepsize is obtained, the variable is updated as follows:

$$
\begin{aligned}
\mathbf{X}^{t+1} & =\mathbf{X}^{t}+\gamma^{t}\left(\mathbb{B}_{X} \mathbf{Z}^{t}-\mathbf{X}^{t}\right), \\
\mathbf{S}^{t+1} & =\mathbf{S}^{t}+\gamma^{t}\left(\mathbb{B}_{S} \mathbf{Z}^{t}-\mathbf{S}^{t}\right) .
\end{aligned}
$$

We name the proposed iterative procedure (41)-(43) as the SoftThresholding with Exact Line search Algorithm (STELA). The sequence $\left\{\mathbf{X}^{t}, \mathbf{S}^{t}\right\}_{t}$ generated by STELA converges to a stationary point of (39) in the sense specified in Theorem 3.

Remark 4. The expression of $\nabla g^{-}\left(\mathbf{X}^{t}\right)$ in (41) given by

$$
\nabla g^{-}\left(\mathbf{X}^{t}\right)=\nabla\left(\lambda \kappa_{0} \sum_{n=1}^{N} \sigma_{n}(\mathbf{X})\right)-\nabla\left(\lambda \sum_{n=1}^{N} \kappa\left(\sigma_{n}(\mathbf{X})\right)\right)
$$

Note that

$$
\nabla\left(\sum_{n=1}^{N} \kappa\left(\sigma_{n}(\mathbf{X})\right)\right)=\mathbf{U} \operatorname{diag}\left(\left[\nabla \kappa\left(D_{11}\right) \ldots \nabla \kappa\left(D_{N N}\right)\right]\right) \mathbf{V}^{T},
$$

where $\mathbf{X}=\mathbf{U D V}^{T}$ (with $D_{n n}=\sigma_{n}(\mathbf{X})$ ) is the SVD of $\mathbf{X}[33$, Lemma 22]. Note that $\nabla\left(\sum_{n=1}^{N} \sigma_{n}(\mathbf{X})\right)$ can be derived from the above expression by setting $\kappa(x)=x$.

On the comparison with DC programming. The objective function in (39) is a difference of convex (DC) functions $f(\mathbf{X}, \mathbf{S})+$ $g^{+}(\mathbf{X}, \mathbf{S})$ and $g^{-}(\mathbf{X})$, and it can also be solved by the standard MM algorithm, which iterates as follows:

$$
\begin{aligned}
\left(\mathbb{B}_{X} \mathbf{Z}^{t}, \mathbb{B}_{S} \mathbf{Z}^{t}\right) & =\underset{\mathbf{X}, \mathbf{S}}{\arg \min }\left\{\begin{array}{l}
f(\mathbf{X}, \mathbf{S})-\left\langle\mathbf{X}-\mathbf{X}^{t}, \nabla g^{-}\left(\mathbf{X}^{t}\right)\right\rangle \\
+g^{+}(\mathbf{X}, \mathbf{S})
\end{array}\right\}, \\
\left(\mathbf{X}^{t+1}, \mathbf{S}^{t+1}\right) & =\left(\mathbb{B}_{X} \mathbf{Z}^{t}, \mathbb{B}_{S} \mathbf{Z}^{t}\right) .
\end{aligned}
$$

This iterative procedure can be interpreted as a special case of the proposed SCA algorithm by choosing in (40) the following approximate function

$$
\tilde{f}\left(\mathbf{X}, \mathbf{S} ; \mathbf{Z}^{t}\right)=f(\mathbf{X}, \mathbf{S}),
$$

and setting the stepsize in (43) to 1 . The convergence of the MM algorithm (44) can be established from the proposed SCA framework as Assumptions (A1)-(A6) are satisfied. However, the MM algorithm (44) has a much higher complexity as it is a two-layer algorithm: the approximate problem in (44a) does not have a closed-form solution and must be solved iteratively. By comparison, the proposed SCA framework is flexible in the sense that we can design the approximate function that preserves the problem structure (namely, the partial convexity in (40)) but is still easy to optimize. Although the line search in (42) is needed, it has a simple closed-form expression and the computational complexity is thus very low.

\section{Sparse Subspace Clustering Through Capped $\ell_{1}$-Norm MiNIMIZATION}

In this section, we consider the sparse subspace clustering problem through the capped $\ell_{1}$-norm minimization introduced in Sec. II-B:

$$
\underset{\mathbf{x}}{\operatorname{minimize}} \frac{1}{2}\|\mathbf{A x}-\mathbf{b}\|_{2}^{2}+\mu \sum_{k=1}^{K} \min \left(\left|x_{k}\right|, \theta\right),
$$

or more compactly,

$$
\underset{\mathbf{x}}{\operatorname{minimize}} \frac{1}{2}\|\mathbf{A} \mathbf{x}-\mathbf{b}\|_{2}^{2}+\mu\|\min (|\mathbf{x}|, \theta \mathbf{1})\|_{1} .
$$




\begin{abstract}
Algorithm 3 STELA: The proposed parallel BCD algorithm with exact line search for the capped $\ell_{1}$-norm minimization problem (45) Data: $t=0, \mathbf{x}^{0}$ (arbitrary but fixed, e.g., $\mathbf{x}^{0}=\mathbf{0}$ ), stop criterion $\delta$. S1: Compute $\mathbb{B} \mathbf{x}^{t}$ according to (14).

S2: Determine the stepsize $\gamma^{t}$ by the exact line search (21).

S3: Update $\mathrm{x}^{t+1}$ according to (17).

S4: If $\left|\left(\mathbb{B} \mathbf{x}^{t}-\mathbf{x}^{t}\right)^{T}\left(\nabla f\left(\mathbf{x}^{t}\right)-\boldsymbol{\xi}^{-}\left(\mathbf{x}^{t}\right)\right)+g^{+}\left(\mathbb{B} \mathbf{x}^{t}\right)-g^{+}\left(\mathbf{x}^{t}\right)\right| \leq \delta$, STOP; otherwise $t \leftarrow t+1$ and go to S1.
\end{abstract}

It is shown in [27] that problem (45) is a special case of (2) by setting

$$
\begin{aligned}
f(\mathbf{x}) & \triangleq \frac{1}{2}\|\mathbf{A} \mathbf{x}-\mathbf{b}\|_{2}^{2}, \\
g^{+}(\mathbf{x}) & \triangleq \mu\|\mathbf{x}\|_{1}, \\
g^{-}(\mathbf{x}) & \triangleq \mu\|\mathbf{x}\|_{1}-\mu\|\min (|\mathbf{x}|, \theta \mathbf{1})\|_{1} .
\end{aligned}
$$

Since $f$ is convex, we adopt the best-response type approximate function: the approximate function consists of $K$ component functions, and in the $k$-th component function, only the $k$-th element, $x_{k}$, of $\mathbf{x}$ is treated as a variable while other elements $\mathbf{x}_{-k} \triangleq\left(x_{j}\right)_{j \neq k}$ are fixed,

$$
\tilde{f}\left(\mathbf{x} ; \mathbf{x}^{t}\right)=\frac{1}{2} \sum_{k=1}^{K} f\left(x_{k}, \mathbf{x}_{-k}^{t}\right)=\frac{1}{2} \sum_{k=1}^{K}\left\|\mathbf{a}_{k} x_{k}+\sum_{j \neq k} \mathbf{a}_{j} x_{j}^{t}-\mathbf{b}\right\|_{2}^{2} .
$$

To obtain the update direction, we solve the approximate problem

$$
\begin{aligned}
\mathbb{B}^{t} & =\underset{\mathbf{x}}{\arg \min }\left\{\tilde{f}\left(\mathbf{x} ; \mathbf{x}^{t}\right)-\left(\mathbf{x}-\mathbf{x}^{t}\right) \boldsymbol{\xi}^{-}\left(\mathbf{x}^{t}\right)+g^{+}(\mathbf{x})\right\} \\
& =\mathbf{d}\left(\mathbf{A}^{T} \mathbf{A}\right)^{-1} \circ \mathcal{S}_{\mu \mathbf{1}}\left(\mathbf{r}\left(\mathbf{x}^{t}, \boldsymbol{\xi}^{-}\left(\mathbf{x}^{t}\right)\right)\right),
\end{aligned}
$$

where

$$
\begin{aligned}
\mathbf{r}\left(\mathbf{x}^{t}, \boldsymbol{\xi}^{-}\left(\mathbf{x}^{t}\right)\right) & \triangleq \mathbf{d}\left(\nabla^{2} f\left(\mathbf{x}^{t}\right)\right) \circ \mathbf{x}^{t}+\boldsymbol{\xi}^{-}\left(\mathbf{x}^{t}\right)-\nabla f\left(\mathbf{x}^{t}\right) \\
& =\mathbf{d}\left(\mathbf{A}^{T} \mathbf{A}\right) \circ \mathbf{x}^{t}+\boldsymbol{\xi}^{-}\left(\mathbf{x}^{t}\right)-\mathbf{A}^{T}\left(\mathbf{A} \mathbf{x}^{t}-\mathbf{b}\right),
\end{aligned}
$$

$\mathbf{d}(\mathbf{X})$ is the diagonal vector of $\mathbf{X}, \mathcal{S}_{\mathbf{a}}(\mathbf{b}) \triangleq[\mathbf{b}-\mathbf{a}]^{+}-[-\mathbf{b}-\mathbf{a}]^{+}$is the soft-thresholding operator, and the subgradient of $g^{-}(\mathbf{x})$ defined in (31) is $\boldsymbol{\xi}^{-}(\mathbf{x})=\left(\xi_{k}^{-}\left(x_{k}\right)\right)_{k=1}^{K}$ with

$$
\xi_{k}^{-}\left(x_{k}\right)= \begin{cases}\mu, & \text { if } x_{k} \geq \theta \\ -\mu, & \text { if } x_{k} \leq-\theta \\ 0, & \text { otherwise }\end{cases}
$$

or more compactly,

$$
\boldsymbol{\xi}^{-}(\mathbf{x})=\frac{1}{2} \mu(\operatorname{sign}(\mathbf{x}-\boldsymbol{\theta})-\operatorname{sign}(-\mathbf{x}-\boldsymbol{\theta})) .
$$

Given the update direction $\mathbb{B} \mathbf{x}^{t}-\mathbf{x}^{t}$, we calculate the stepsize $\gamma^{t}$ according to the proposed exact line search (21), which can be performed in a simple closed-form expression:

$$
\begin{aligned}
\gamma^{t} & =\underset{0 \leq \gamma \leq 1}{\arg \min }\left\{\begin{array}{l}
f\left(\mathbf{x}^{t}+\gamma\left(\mathbb{B} \mathbf{x}^{t}-\mathbf{x}^{t}\right)\right) \\
+\gamma\left(g^{+}\left(\mathbb{B} \mathbf{x}^{t}\right)-g^{+}\left(\mathbf{x}^{t}\right)-\left(\mathbb{B} \mathbf{x}^{t}-\mathbf{x}^{t}\right)^{T} \boldsymbol{\xi}^{-}\left(\mathbf{x}^{t}\right)\right)
\end{array}\right\} \\
& =\left[\frac{\left(\boldsymbol{\xi}^{-}\left(\mathbf{x}^{t}\right)-\mathbf{A}^{T}\left(\mathbf{A} \mathbf{x}^{t}-\mathbf{b}\right)\right)^{T}\left(\mathbb{B} \mathbf{x}^{t}-\mathbf{x}^{t}\right)-\mu\left(\left\|\mathbb{B} \mathbf{x}^{t}\right\|_{1}-\left\|\mathbf{x}^{t}\right\|_{1}\right)}{\left(\mathbf{A}\left(\mathbb{B} \mathbf{x}^{t}-\mathbf{x}^{t}\right)\right)^{T}\left(\mathbf{A}\left(\mathbb{B} \mathbf{x}^{t}-\mathbf{x}^{t}\right)\right)}\right]_{0}^{1} .
\end{aligned}
$$

The proposed update (48)-(49) are summarized in Algorithm 3 and we name it as Soft-Thresholding with Exact Line search Algorithm (STELA). It has several attractive features:

- i) low complexity, as the approximate function is chosen such that its minimum can be obtained in closed-form expressions and the proposed algorithm thus has a single layer. Besides this, the stepsize can also be computed by closed-form expressions;

- ii) fast convergence, as all elements are updated in parallel, the approximate function is of a best-response type, and the stepsize is based on the exact line search;

- iii) guaranteed convergence, as $\tilde{f}\left(\mathbf{x} ; \mathbf{x}^{t}\right)$ in (47) is strongly convex and Assumptions (A4)-(A5) are satisfied.

Compared with state-of-the-art algorithms proposed for problem (45), we remark that

- feature i) is an advantage over the traditional MM method [27];

- feature ii) is an advantage over the algorithms [29, 30] with a proximal type approximation;

- feature iii) is an advantage over the standard SCA framework for convex regularization functions $[6,15,16]$.

On the comparison with the proximal MM method [29]. The proximal type algorithm proposed in [29] is essentially a MM method, because the variable is updated by

$\mathbf{x}^{t+1}=\underset{\mathbf{x} \in \mathcal{X}}{\arg \min }\left\{\begin{array}{l}f\left(\mathbf{x}^{t}\right)+\nabla f\left(\mathbf{x}^{t}\right)^{T}\left(\mathbf{x}-\mathbf{x}^{t}\right)+\frac{c^{t}}{2}\left\|\mathbf{x}-\mathbf{x}^{t}\right\|^{2} \\ +g^{+}(\mathbf{x})-g^{-}(\mathbf{x})\end{array}\right\}$,

with $c^{t}>L_{\nabla f}$, while the objective function in (50) is a global upper bound of $h(\mathbf{x})$ in view of the descent lemma [17, Prop. A.24]. When the value of $L_{\nabla f}$ is not known, $c^{t}$ is estimated iteratively: for some constants $0<\alpha<1$ and $0<\beta<1$, set $\mathbf{x}^{t+1}=\mathbf{x}^{\star}\left(\beta^{m_{t}}\right)$, where $\mathbf{x}^{\star}\left(\beta^{m}\right)$ is defined as

$\mathbf{x}^{\star}\left(\beta^{m}\right) \triangleq \underset{\mathbf{x}}{\arg \min }\left\{\begin{array}{l}f\left(\mathbf{x}^{t}\right)+\nabla f\left(\mathbf{x}^{t}\right)^{T}\left(\mathbf{x}-\mathbf{x}^{t}\right)+\frac{1}{2 \beta^{m}}\left\|\mathbf{x}-\mathbf{x}^{t}\right\|^{2} \\ +g^{+}(\mathbf{x})-g^{-}(\mathbf{x})\end{array}\right\}$

and $m_{t}$ is the smallest nonnegative integer such that $h\left(\mathbf{x}^{\star}\left(\beta^{m_{t}}\right)\right)-$ $h\left(\mathbf{x}^{t}\right) \leq-\alpha / 2 \beta^{m_{t}}\left\|\mathbf{x}^{\star}\left(\beta^{m_{t}}\right)-\mathbf{x}^{t}\right\|^{2}$. As a result, $\mathbf{x}^{\star}\left(\beta^{m}\right)$ must be evaluated repeatedly for $m_{t}$ times, namely, $m=0,1, \ldots, m_{t}$. This is however not necessary in the proposed algorithm STELA, because computing the descent direction and the stepsize according to (48) and (49) does not depend on any unknown parameters. Furthermore, (51) may not be easy to solve for a general $g^{-}(\mathbf{x})$ except for some specific choices studied in [29].

\section{Numerical Simulations}

In our numerical simulations the dimension of $\mathbf{A}$ is $10000 \times 50000$ : all of its elements are generated randomly by the normal distribution $\mathcal{N}(0,1)$, and the rows of $\mathbf{A}$ are normalized to have a unit $\ell_{2}$-norm. The density (the proportion of nonzero elements) of the sparse vector $\mathbf{x}_{\text {true }}$ is 0.1 . The vector $\mathbf{b}$ is generated as $\mathbf{b}=\mathbf{A} \mathbf{x}_{\text {true }}+\mathbf{e}$ where $\mathbf{e}$ is drawn from an i.i.d. Gaussian distribution with mean 0 and variance $10^{-4}$. The regularization parameter $\mu$ is set to $\mu=0.1\left\|\mathbf{A}^{T} \mathbf{b}\right\|_{\infty}$, which allows $\mathbf{x}_{\text {true }}$ to be recovered to a high accuracy [39], and the parameter $\theta$ in the capped $\ell_{1}$-norm is set to 1 .

We compare the proposed algorithm STELA with the classic MM method [27] and the proximal MM algorithm [29]. The comparison is made in terms of CPU time that is required until the maximum number of iterations (100 for STELA and the proximal MM algorithm and 10 for the classic MM method) is reached. The running time consists of both the initialization stage required for preprocessing (represented by a flat curve) and the formal stage in which the iterations are carried out. For example, in STELA, $\mathbf{d}\left(\mathbf{A}^{T} \mathbf{A}\right)$ is computed in the initialization stage since it is required in the iterative variable update in the formal stage, cf. (48). The upper bound function in the classic MM method, cf. (11), is minimized by STELA for $\ell_{1}$ norm (with a warm start that sets the optimal point of the previous 


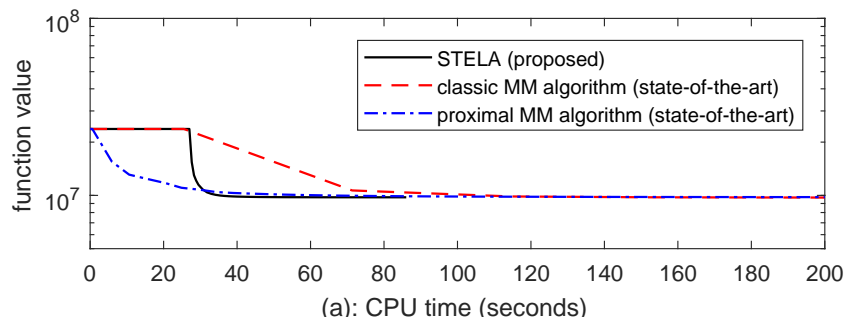

a): CPU time (seconds)

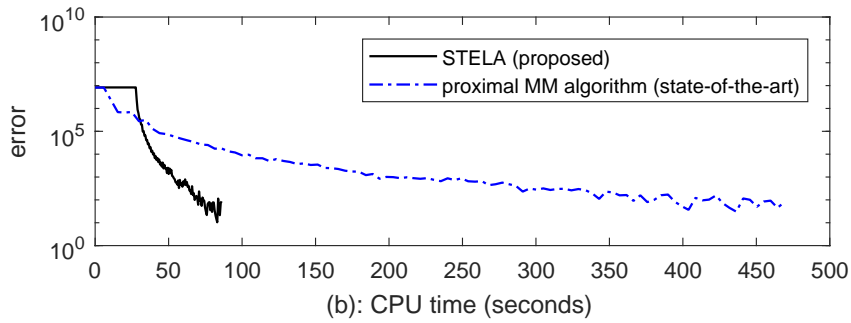

Figure 3. Capped $\ell_{1}$-norm minimization: achieved function value $h\left(\mathbf{x}^{t}\right)$ and error $h\left(\mathbf{x}^{t-1}\right)-h\left(\mathbf{x}^{t}\right)$ versus CPU time (in seconds).

iteration as the initial point of the current iteration [27, Sec. II-D]), which was presented in [6, Sec. IV-III]. All algorithms have the same initial point, $\mathbf{x}^{0}=\mathbf{0}$. The simulation results are averaged over 20 instances.

The achieved function value $h\left(\mathbf{x}^{t}\right)$ and error $h\left(\mathbf{x}^{t-1}\right)-h\left(\mathbf{x}^{t}\right)$ versus the CPU time (in seconds) is plotted in Fig. 3 (a) and 3 (b), respectively. We see from Fig. 3 (a) that all algorithms converge to the same value. Furthermore, the initialization stage of STELA is much longer than that of the proximal $\mathrm{MM}$ algorithm, because computing $\mathbf{d}\left(\mathbf{A}^{T} \mathbf{A}\right)$, the diagonal vector of $\mathbf{A}^{T} \mathbf{A}$, is computationally expensive, especially when the dimension of $\mathbf{A}$ is large. Nevertheless, in the formal stage, the convergence speed of STELA is much faster than the proximal MM algorithm, and this is mainly due to the use of the best-response type approximate function (47), and more specifically, the use of $\mathbf{d}\left(\mathbf{A}^{T} \mathbf{A}\right)$, cf. (48), which represents partial second order information of the function $f$ in (45) (note that $\left.\nabla^{2} f(\mathbf{x})=\mathbf{A}^{T} \mathbf{A}\right)$. We see from Fig. 3 (b) that the long initialization stage is compensated by the fast convergence speed in the formal stage. We mention for the paper's completeness that $\mathbf{d}\left(\mathbf{A}^{T} \mathbf{A}\right)$ can be calculated analytically in some applications, e.g., when $\mathbf{A}$ is a Vandermonde or constant modulus matrix.

We see from Fig. 3 (a) that the major complexity of the classic MM method lies in the first few iterations, as the complexity of late iterations are notably reduced by a good initialization thanks to the warm start. The most notable difference between the MM method and the STELA is that the upper bound function is only approximately minimized in the STELA, and this leads to a significant reduction in the computational complexity. Using the approximate function is also beneficial when the upper bound function $\bar{h}\left(\mathbf{x} ; \mathbf{x}^{t}\right)$ is not easy to minimize, e.g., $f(\mathbf{x})$ is nonconvex.

\section{CONCLUding REMARKS}

In this paper, we have proposed a successive convex approximation framework for sparse signal estimation where the nonsmooth nonconvex regularization function is nonconvex and can be written as the difference of two convex functions. The proposed procedure is to apply the standard successive convex approximation for convex regularization functions to an upper bound of the original objective function that can be obtained following the standard MM method. This procedure also facilitates the design of low-complexity line search schemes which are carried out over a differentiable function. The proposed framework is flexible and it leads to algorithms that exploit the problem structure and have a low complexity. Customizing the general framework for the example applications in network anomaly detection and sparse subspace clustering, the proposed algorithm STELA is a parallel BCD algorithm with exact line search and it has several attractive features, illustrated both theoretically and numerically: i) fast convergence due to the best-response type approximation and the line search for stepsize calculation; ii) low complexity as both the optimal point of the approximate function and the exact line search have closed-form expressions; and iii) guaranteed convergence to a stationary point.

\section{APPENDIX A}

PROOF OF PROPOSITIONS 1 AND 2

Proof of Proposition 1: Since the approximate problem (13) is convex, $\mathbb{B} \mathbf{x}^{t}$ is a globally optimal point of (13) and

$$
\tilde{h}\left(\mathbb{B} \mathbf{x}^{t} ; \mathbf{x}^{t}\right)=\min _{\mathbf{x} \in \mathcal{X}} \tilde{h}\left(\mathbf{x} ; \mathbf{x}^{t}\right) \leq \tilde{h}\left(\mathbf{x}^{t} ; \mathbf{x}^{t}\right) .
$$

We discuss the two possibilities separately, namely,

$$
\text { i): } \tilde{h}\left(\mathbb{B} \mathbf{x}^{t} ; \mathbf{x}^{t}\right)=\tilde{h}\left(\mathbf{x}^{t} ; \mathbf{x}^{t}\right),
$$

or

$$
\text { ii): } \tilde{h}\left(\mathbb{B} \mathbf{x}^{t} ; \mathbf{x}^{t}\right)<\tilde{h}\left(\mathbf{x}^{t} ; \mathbf{x}^{t}\right) \text {. }
$$

i) $\tilde{h}\left(\mathbb{B} \mathbf{x}^{t} ; \mathbf{x}^{t}\right)=\tilde{h}\left(\mathbf{x}^{t} ; \mathbf{x}^{t}\right)$. We show that $\tilde{h}\left(\mathbb{B} \mathbf{x}^{t} ; \mathbf{x}^{t}\right)=\tilde{h}\left(\mathbf{x}^{t} ; \mathbf{x}^{t}\right)$ is equivalent to $\mathbf{x}^{t}$ being a stationary point of (2).

If $\tilde{h}\left(\mathbb{B} \mathbf{x}^{t} ; \mathbf{x}^{t}\right)=\tilde{h}\left(\mathbf{x}^{t} ; \mathbf{x}^{t}\right)$, then $\mathbf{x}^{t} \in \mathcal{S}\left(\mathbf{x}^{t}\right)$ :

$$
\mathbf{x}^{t} \in \underset{\mathbf{x} \in \mathcal{X}}{\arg \min } \tilde{h}\left(\mathbf{x} ; \mathbf{x}^{t}\right),
$$

and it must satisfy the first-order optimality condition: for some $\boldsymbol{\xi}^{+}\left(\mathbf{x}^{t}\right)$

$$
\left(\mathbf{x}-\mathbf{x}^{t}\right)\left(\nabla \tilde{f}\left(\mathbf{x}^{t} ; \mathbf{x}^{t}\right)+\boldsymbol{\xi}^{+}\left(\mathbf{x}^{t}\right)-\boldsymbol{\xi}^{-}\left(\mathbf{x}^{t}\right)\right) \geq 0, \forall \mathbf{x} .
$$

This is exactly the first-order optimality condition of problem (2) after replacing $\nabla \tilde{f}\left(\mathbf{x}^{t} ; \mathbf{x}^{t}\right)$ by $\nabla f\left(\mathbf{x}^{t}\right)$ in view of Assumption (A3) on the gradient consistency. Therefore, $\mathbf{x}^{t}$ is a stationary point of (2).

Reversely, if $\mathbf{x}^{t}$ is a stationary point of (2), then it satisfies the first-order optimality condition: for some $\boldsymbol{\xi}^{+}\left(\mathbf{x}^{t}\right)$ and $\boldsymbol{\xi}^{-}\left(\mathbf{x}^{t}\right)$,

$$
\left(\mathbf{x}-\mathbf{x}^{t}\right)\left(\nabla f\left(\mathbf{x}^{t}\right)+\boldsymbol{\xi}^{+}\left(\mathbf{x}^{t}\right)-\boldsymbol{\xi}^{-}\left(\mathbf{x}^{t}\right)\right) \geq 0, \forall \mathbf{x} .
$$

By assumption (A3) on the gradient consistency, the above condition is equivalent to

$$
\left(\mathbf{x}-\mathbf{x}^{t}\right)\left(\nabla \tilde{f}\left(\mathbf{x}^{t} ; \mathbf{x}^{t}\right)+\boldsymbol{\xi}^{+}\left(\mathbf{x}^{t}\right)-\boldsymbol{\xi}^{-}\left(\mathbf{x}^{t}\right)\right) \geq 0, \forall \mathbf{x} .
$$

Since problem (13) is convex, the above condition implies that $\mathbf{x}^{t}$ is a globally optimal point of (14) and $\tilde{h}\left(\mathbf{x}^{t} ; \mathbf{x}^{t}\right)=\min _{\mathbf{x} \in \mathcal{X}} \tilde{h}\left(\mathbf{x} ; \mathbf{x}^{t}\right)$.

ii) $\tilde{h}\left(\mathbb{B} \mathbf{x}^{t} ; \mathbf{x}^{t}\right)<\tilde{h}\left(\mathbf{x}^{t} ; \mathbf{x}^{t}\right)$. We remark that problem (13) is convex and equivalent to the following problem

$$
\begin{array}{ll}
\underset{\mathbf{x}, y}{\operatorname{minimize}} & \tilde{f}\left(\mathbf{x} ; \mathbf{x}^{t}\right)-\left(\mathbf{x}-\mathbf{x}^{t}\right)^{T} \boldsymbol{\xi}^{-}\left(\mathbf{x}^{t}\right)+y \\
\text { subject to } & \mathbf{x} \in \mathcal{X}, g^{+}(\mathbf{x}) \leq y .
\end{array}
$$

The equivalence between (12) and (54) is in the sense that $\mathbb{B} \mathbf{x}^{t}$ defined in (14) is the optimal $\mathbf{x}$ of (54), and the optimal $y$ of (54), denoted as $y^{\star}\left(\mathbf{x}^{t}\right)$, is given by $y^{\star}\left(\mathbf{x}^{t}\right)=g^{+}\left(\mathbb{B} \mathbf{x}^{t}\right)$. If $\tilde{h}\left(\mathbb{B} \mathbf{x}^{t} ; \mathbf{x}^{t}\right)<$ 


$$
\begin{aligned}
& \tilde{h}\left(\mathbf{x}^{t} ; \mathbf{x}^{t}\right), \text { then } \\
& \begin{aligned}
\tilde{h}\left(\mathbb{B} \mathbf{x}^{t} ; \mathbf{x}^{t}\right) & =\tilde{f}\left(\mathbb{B} \mathbf{x}^{t} ; \mathbf{x}^{t}\right)-\left(\mathbb{B} \mathbf{x}^{t}-\mathbf{x}^{t}\right)^{T} \boldsymbol{\xi}^{-}\left(\mathbf{x}^{t}\right)+g^{+}\left(\mathbb{B} \mathbf{x}^{t}\right) \\
& \stackrel{(a)}{=} \tilde{f}\left(\mathbb{B} \mathbf{x}^{t} ; \mathbf{x}^{t}\right)-\left(\mathbb{B} \mathbf{x}^{t}-\mathbf{x}^{t}\right)^{T} \boldsymbol{\xi}^{-}\left(\mathbf{x}^{t}\right)+y^{\star}\left(\mathbf{x}^{t}\right) \\
& <\tilde{h}\left(\mathbf{x}^{t} ; \mathbf{x}^{t}\right) \\
& =\tilde{f}\left(\mathbf{x}^{t} ; \mathbf{x}^{t}\right)-\left(\mathbf{x}^{t}-\mathbf{x}^{t}\right)^{T} \boldsymbol{\xi}^{-}\left(\mathbf{x}^{t}\right)+g^{+}\left(\mathbf{x}^{t}\right) \\
& \stackrel{(b)}{\leq} \tilde{f}\left(\mathbf{x}^{t} ; \mathbf{x}^{t}\right)-\left(\mathbf{x}^{t}-\mathbf{x}^{t}\right)^{T} \boldsymbol{\xi}^{-}\left(\mathbf{x}^{t}\right)+y^{t},
\end{aligned}
\end{aligned}
$$

where the equality (a) follows from the fact that $y^{\star}\left(\mathbf{x}^{t}\right)=g^{+}\left(\mathbb{B} \mathbf{x}^{t}\right)$, and the inequality $(b)$ follows from the fact that $y^{t} \geq g^{+}\left(\mathbf{x}^{t}\right)$ in view of the constraint in (54). Since $y^{t}$ does not appear in (54), we set without loss of generality $y^{t}=g^{+}\left(\mathbf{x}^{t}\right)$.

The objective function of (54) is convex and differentiable, and thus also pseudoconvex [6, Figure 1]. From the definition of pseudoconvex functions that

$$
\begin{aligned}
& \tilde{f}\left(\mathbb{B} \mathbf{x}^{t} ; \mathbf{x}^{t}\right)-\left(\mathbb{B} \mathbf{x}^{t}-\mathbf{x}^{t}\right)^{T} \boldsymbol{\xi}^{-}\left(\mathbf{x}^{t}\right)+y^{\star}\left(\mathbf{x}^{t}\right) \\
< & \tilde{f}\left(\mathbf{x}^{t} ; \mathbf{x}^{t}\right)-\left(\mathbf{x}^{t}-\mathbf{x}^{t}\right)^{T} \boldsymbol{\xi}^{-}\left(\mathbf{x}^{t}\right)+y^{t}
\end{aligned}
$$

implies

$$
\left(\mathbb{B} \mathbf{x}^{t}-\mathbf{x}^{t}\right)^{T}\left(\nabla \tilde{f}\left(\mathbf{x}^{t} ; \mathbf{x}^{t}\right)-\boldsymbol{\xi}^{-}\left(\mathbf{x}^{t}\right)\right)+y^{\star}\left(\mathbb{B} \mathbf{x}^{t}\right)-y^{t}<0,
$$

which is equivalent to the following inequality after replacing $\nabla \tilde{f}\left(\mathbf{x}^{t} ; \mathbf{x}^{t}\right)$ by $\nabla f\left(\mathbf{x}^{t}\right)$ in view of Assumption (A3) on the gradient consistency:

$$
\left(\mathbb{B} \mathbf{x}^{t}-\mathbf{x}^{t}\right)^{T}\left(\nabla f\left(\mathbf{x}^{t}\right)-\boldsymbol{\xi}^{-}\left(\mathbf{x}^{t}\right)\right)+y^{\star}\left(\mathbb{B} \mathbf{x}^{t}\right)-y^{t}<0,
$$

where $y^{\star}\left(\mathbb{B} \mathbf{x}^{t}\right)=g^{+}\left(\mathbb{B} \mathbf{x}^{t}\right)$ and $y^{t}=g^{+}\left(\mathbf{x}^{t}\right)$. Therefore, we readily obtain the inequality in (16) and the proof of Proposition 1 is thus completed.

Proof of Proposition 2: We define

$$
l\left(\mathbf{x}, y ; \mathbf{x}^{t}\right) \triangleq f(\mathbf{x})-\left(\mathbf{x}-\mathbf{x}^{t}\right)^{T} \boldsymbol{\xi}^{-}\left(\mathbf{x}^{t}\right)+y .
$$

We can see that $\nabla_{\mathbf{x}} l\left(\mathbf{x}, y ; \mathbf{x}^{t}\right)=\nabla f(\mathbf{x})-\boldsymbol{\xi}^{-}\left(\mathbf{x}^{t}\right)$ and $\nabla_{y} l\left(\mathbf{x}, y ; \mathbf{x}^{t}\right)=1$. Then the inequality (55) can be rewritten as

$$
\begin{aligned}
0 & >\left(\mathbb{B} \mathbf{x}^{t}-\mathbf{x}^{t}\right)^{T} \nabla_{\mathbf{x}} l\left(\mathbf{x}^{t}, y^{t} ; \mathbf{x}^{t}\right)+\left(y^{\star}\left(\mathbb{B} \mathbf{x}^{t}\right)-y^{t}\right) \nabla_{y} l\left(\mathbf{x}^{t}, y^{t} ; \mathbf{x}^{t}\right) \\
& =\left(\mathbb{B} \mathbf{x}^{t}-\mathbf{x}^{t}, y^{\star}\left(\mathbf{x}^{t}\right)-y^{t}\right)^{T} \nabla l\left(\mathbf{x}^{t}, y^{t} ; \mathbf{x}^{t}\right)
\end{aligned}
$$

From the above inequality we can claim that $\left(\mathbb{B} \mathbf{x}^{t}, y^{\star}\left(\mathbf{x}^{t}\right)\right)-\left(\mathbf{x}^{t}, y^{t}\right)$ is a descent direction of the function $l\left(\mathbf{x}, y ; \mathbf{x}^{t}\right)=f(\mathbf{x})-(\mathbf{x}-$ $\left.\mathbf{x}^{t}\right)^{T} \boldsymbol{\xi}^{-}\left(\mathbf{x}^{t}\right)+y$ at the point $\left(\mathbf{x}^{t}, y^{t}\right)$.

The proposed exact line search (21) is equivalent to applying the standard exact line search to the differentiable function $l\left(\mathbf{x}, y ; \mathbf{x}^{t}\right)$ along the direction $\left(\mathbb{B} \mathbf{x}^{t}, y^{\star}\left(\mathbf{x}^{t}\right)\right)-\left(\mathbf{x}^{t}, y^{t}\right)$ :

$$
\gamma^{t}=\underset{0 \leq \gamma \leq 1}{\arg \min }\left\{\begin{array}{l}
f\left(\mathbf{x}^{t}+\gamma\left(\mathbb{B} \mathbf{x}^{t}-\mathbf{x}^{t}\right)\right) \\
-\left(\mathbf{x}^{t}+\gamma\left(\mathbb{B} \mathbf{x}^{t}-\mathbf{x}^{t}\right)-\mathbf{x}^{t}\right)^{T} \boldsymbol{\xi}^{-}\left(\mathbf{x}^{t}\right) \\
+y^{t}+\gamma\left(y^{\star}\left(\mathbf{x}^{t}\right)-y^{t}\right) .
\end{array}\right\} .
$$

Therefore the existence of a $\gamma^{t} \in(0,1]$ is guaranteed according to [40, 8.2.1].

Similarly, the proposed successive line search is equivalent to applying the standard successive line search to the differentiable function $l\left(\mathbf{x}, y ; \mathbf{x}^{t}\right)$ along the direction $\left(\mathbb{B} \mathbf{x}^{t}, y^{\star}\left(\mathbf{x}^{t}\right)\right)-\left(\mathbf{x}^{t}, y^{t}\right)$ :

$$
\begin{aligned}
& l\left(\mathbf{x}^{t}+\beta^{m}\left(\mathbb{B} \mathbf{x}^{t}-\mathbf{x}^{t}\right), y^{t}+\beta^{m}\left(y^{\star}\left(\mathbf{x}^{t}\right)-y^{t}\right) ; \mathbf{x}^{t}\right) \\
\leq & l\left(\mathbf{x}^{t}, y^{t} ; \mathbf{x}^{t}\right)+\alpha \beta^{m}\left(\mathbb{B} \mathbf{x}^{t}-\mathbf{x}^{t}, y^{\star}\left(\mathbf{x}^{t}\right)-y^{t}\right)^{T} \nabla l\left(\mathbf{x}^{t}, y^{t} ; \mathbf{x}^{t}\right) .
\end{aligned}
$$

The proof of Proposition 2 is thus completed.

\section{APPENDIX B}

PROOF OF THEOREM 3

Proof: Similar to [6, Theorem 1], the key of the proof is to show that $\mathbb{B} \mathbf{x}$ is a closed mapping [41], i.e., if $\lim _{t \rightarrow \infty} \mathbf{x}^{t}=\mathbf{x}$ and $\lim _{t \rightarrow \infty} \mathbb{B} \mathbf{x}^{t}=\mathbf{y}$, then $\mathbb{B} \mathbf{x} \in \mathcal{S}(\mathbf{x})$. The key difference is that the objective function $h$ in (2) is nondifferentiable.

Since $\mathbb{B} \mathbf{x}^{t}$ is the optimal point of (13), it satisfies the first-order optimality condition:

$$
\left(\mathbf{x}-\mathbb{B} \mathbf{x}^{t}\right)^{T}\left(\nabla \tilde{f}\left(\mathbb{B} \mathbf{x}^{t} ; \mathbf{x}^{t}\right)-\boldsymbol{\xi}^{-}\left(\mathbf{x}^{t}\right)+\boldsymbol{\xi}^{+}\left(\mathbf{x}^{t}\right)\right) \geq 0, \forall \mathbf{x} \in \mathcal{X} .
$$

If (52) is true, then $\mathbf{x}^{t} \in \mathcal{S}\left(\mathbf{x}^{t}\right)$ and it is a stationary point of (2) according to Proposition 1 (i). Besides, it follows from (2) (with $\mathbf{x}=$ $\mathbb{B} \mathbf{x}^{t}$ and $\left.\mathbf{y}=\mathbf{x}^{t}\right)$ that $\left(\mathbb{B} \mathbf{x}^{t}-\mathbf{x}^{t}\right)^{T}\left(\nabla f\left(\mathbf{x}^{t}\right)-\boldsymbol{\xi}^{-}\left(\mathbf{x}^{t}\right)+\boldsymbol{\xi}^{+}\left(\mathbf{x}^{t}\right)\right) \geq 0$. Note that equality is actually achieved, i.e.,

$$
\left(\mathbb{B} \mathbf{x}^{t}-\mathbf{x}^{t}\right)^{T}\left(\nabla f\left(\mathbf{x}^{t}\right)-\boldsymbol{\xi}^{-}\left(\mathbf{x}^{t}\right)+\boldsymbol{\xi}^{+}\left(\mathbf{x}^{t}\right)\right)=0
$$

because otherwise $\mathbb{B} \mathbf{x}^{t}-\mathbf{x}^{t}$ would be an ascent direction of $\tilde{h}\left(\mathbf{x} ; \mathbf{x}^{t}\right)$ at $\mathbf{x}=\mathbf{x}^{t}$ and the definition of $\mathbb{B} \mathbf{x}^{t}$ would be contradicted. Then from the definition of the proposed successive line search in (23), we can readily infer that

$$
h\left(\mathbf{x}^{t+1}\right) \leq h\left(\mathbf{x}^{t}\right) .
$$

It is easy to see (57) holds for the exact line search as well.

If (53) is true, $\mathbf{x}^{t}$ is not a stationary point and $\mathbb{B} \mathbf{x}^{t}-\mathbf{x}^{t}$ is a strict descent direction of $h(\mathbf{x})$ at $\mathbf{x}=\mathbf{x}^{t}$ according to Proposition 1 (ii): $h(\mathbf{x})$ is strictly decreased compared with $h\left(\mathbf{x}^{t}\right)$ if $\mathbf{x}$ is updated at $\mathbf{x}^{t}$ along the direction $\mathbb{B} \mathbf{x}^{t}-\mathbf{x}^{t}$. From Proposition 2, the proposed successive line search schemes yield a stepsize $\gamma^{t}$ such that $0<$ $\gamma^{t} \leq 1$ and

$$
h\left(\mathbf{x}^{t+1}\right)=h\left(\mathbf{x}^{t}+\gamma^{t}\left(\mathbb{B} \mathbf{x}^{t}-\mathbf{x}^{t}\right)\right)<h\left(\mathbf{x}^{t}\right) .
$$

This strict decreasing property also holds for the exact line search because it is the stepsize that yields the largest decrease, which is always larger than or equal to that of the successive line search.

We know from (57) and (58) that $\left\{h\left(\mathbf{x}^{t}\right)\right\}$ is a monotonically decreasing sequence and it thus converges. Besides, for any two (possibly different) convergent subsequences $\left\{\mathbf{x}^{t}\right\}_{t \in \mathcal{T}_{1}}$ and $\left\{\mathbf{x}^{t}\right\}_{t \in \mathcal{T}_{2}}$, the following holds:

$$
\lim _{t \rightarrow \infty} h\left(\mathbf{x}^{t}\right)=\lim _{\mathcal{T}_{1} \ni t \rightarrow \infty} h\left(\mathbf{x}^{t}\right)=\lim _{\mathcal{T}_{2} \ni t \rightarrow \infty} h\left(\mathbf{x}^{t}\right) .
$$

Since $h(\mathbf{x})$ is a continuous function, we infer from the preceding equation that

$$
h\left(\lim _{\mathcal{T}_{1} \ni t \rightarrow \infty} \mathbf{x}^{t}\right)=h\left(\lim _{\mathcal{T}_{2} \ni t \rightarrow \infty} \mathbf{x}^{t}\right) .
$$

Now consider any convergent subsequence $\left\{\mathbf{x}^{t}\right\}_{t \in \mathcal{T}}$ with limit point $\mathbf{y}$, i.e., $\lim _{\mathcal{T} \ni t \rightarrow \infty} \mathbf{x}^{t}=\mathbf{y}$. To show that $\mathbf{y}$ is a stationary point, we first assume the contrary: $\mathbf{y}$ is not a stationary point. Since $\tilde{h}\left(\mathbf{x} ; \mathbf{x}^{t}\right)$ is continuous in both $\mathbf{x}$ and $\mathbf{x}^{t}$ by Assumption (A2) and $\left\{\mathbf{B x}^{t}\right\}_{t \in \mathcal{T}}$ is bounded by Assumption (A5), there exists a sequence $\left\{\mathbb{B} \mathbf{x}^{t}\right\}_{t \in \mathcal{T}_{s}}$ with $\mathcal{T}_{s} \subseteq \mathcal{T}$ such that it converges and it follows from the Maximum Theorem in [41, Ch. VI.3] that $\lim _{\mathcal{T}_{s} \ni t \rightarrow \infty} \mathbb{B} \mathbf{x}^{t} \in \mathcal{S}(\mathbf{y})$. Since both $f(\mathbf{x})$ and $\nabla f(\mathbf{x})$ are continuous, applying the Maximum Theorem again implies there is a $\mathcal{T}_{s^{\prime}}$ such that $\mathcal{T}_{s^{\prime}} \subseteq \mathcal{T}_{s}(\subseteq \mathcal{T})$ and $\left\{\mathbf{x}^{t+1}\right\}_{t \in \mathcal{T}_{s^{\prime}}}$ converges to $\mathbf{y}^{\prime}$ defined as $\mathbf{y}^{\prime} \triangleq \mathbf{y}+\rho(\mathbb{B} \mathbf{y}-\mathbf{y})$, where $\rho$ is the stepsize when either the exact or successive line search is applied to $f(\mathbf{y})$ along the direction $\mathbb{B} \mathbf{y}-\mathbf{y}$. Since $\mathbf{y}$ is not a stationary point, it follows from (58) that $h\left(\mathbf{y}^{\prime}\right)<h(\mathbf{y})$, but this would contradict (59). Therefore $\mathbf{y}$ is a stationary point, and the proof is completed. 


\section{REFERENCES}

[1] S. Theodoridis, Machine Learning: A Bayesian and Optimization Perspective, 1st ed. Academic Press, 2015.

[2] A. Beck and M. Teboulle, "A Fast Iterative Shrinkage-Thresholding Algorithm," Society for Industrial and Applied Mathematics Journal on Imaging Sciences, vol. 2, no. 1, pp. 183-202, 2009.

[3] P. Tseng, "Convergence of a Block Coordinate Descent Method for Nondifferentiable Minimization," Journal of Optimization Theory and Applications, vol. 109, no. 3, pp. 475-494, Jun. 2001.

[4] S. Boyd, N. Parikh, E. Chu, B. Peleato, and J. Eckstein, "Distributed Optimization and Statistical Learning via the Alternating Direction Method of Multipliers," Foundations and Trends in Machine Learning, vol. 3, no. 1, 2010

[5] N. Parikh and S. Boyd, "Proximal Algorithms," Foundations and Trends in Optimization, vol. 1, no. 3, pp. 127-239, 2014.

[6] Y. Yang and M. Pesavento, "A Unified Successive Pseudoconvex Approximation Framework," IEEE Transactions on Signal Processing, vol. 65, no. 13, pp. 3313-3328, Jul. 2017

[7] Z. Yang, Z. Wang, H. Liu, Y. C. Eldar, and T. Zhang, "Sparse Nonlinear Regression: Parameter Estimation and Asymptotic Inference," in International Conference on Machine Learning (ICML), 2016.

[8] D. P. Bertsekas and J. N. Tsitsiklis, Parallel and distributed computation: Numerical methods. Prentice Hall, 1989.

[9] M. Razaviyayn, M. Hong, and Z.-Q. Luo, "A Unified Convergence Analysis of Block Successive Minimization Methods for Nonsmooth Optimization," SIAM Journal on Optimization, vol. 23, no. 2, pp. 1126-1153, Jan. 2013

[10] A. Beck and L. Tetruashvili, "On the Convergence of Block Coordinate Descent Type Methods," SIAM Journal on Optimization, vol. 23, no. 4, pp. 2037-2060, Jan. 2013.

[11] S. J. Wright, "Coordinate descent algorithms," Mathematical Programming, vol. 151, no. 1, pp. 3-34, 2015.

[12] M. Mardani, G. Mateos, and G. B. Giannakis, "Dynamic anomalography: Tracking network anomalies via sparsity and low rank," IEEE Journal on Selected Topics in Signal Processing, vol. 7, no. 1, pp. 50-66, Feb. 2013.

[13] K. Slavakis, G. B. Giannakis, and G. Mateos, "Modeling and Optimization for Big Data Analytics: (Statistical) learning tools for our era of data deluge," IEEE Signal Processing Magazine, vol. 31, no. 5 , pp. 18-31, Sep. 2014.

[14] M. Elad, "Why simple shrinkage is still relevant for redundant representations?" IEEE Transactions on Information Theory, vol. 52, no. 12 , pp. 5559-5569, Dec. 2006.

[15] M. Razaviyayn, M. Hong, Z.-Q. Luo, and J.-S. Pang, "Parallel Successive Convex Approximation for Nonsmooth Nonconvex Optimization," in Proceedings of the 27th International Conference on Neural Information Processing Systems, 2014, pp. 1440-1448.

[16] F. Facchinei, G. Scutari, and S. Sagratella, "Parallel Selective Algorithms for Nonconvex Big Data Optimization," IEEE Transactions on Signal Processing, vol. 63, no. 7, pp. 1874-1889, Nov. 2015.

[17] D. P. Bertsekas, Nonlinear programming. Athena Scientific, 1999.

[18] C. Steffens, Y. Yang, and M. Pesavento, "Multidimensional sparse recovery for MIMO channel parameter estimation," European Signal Processing Conference, pp. 66-70, 2016.

[19] M. Mardani, G. Mateos, and G. B. Giannakis, "Decentralized sparsityregularized rank minimization: Algorithms and applications," IEEE Transactions on Signal Processing, vol. 61, no. 21, pp. 5374-5388, Nov 2013.

[20] M. Hong, Z.-Q. Luo, and M. Razaviyayn, "Convergence Analysis of Alternating Direction Method of Multipliers for a Family of Nonconvex Problems," SIAM Journal on Optimization, vol. 26, no. 1, pp. 337-364, Jan. 2016.

[21] B. Jiang, T. Lin, S. Ma, and S. Zhang, "Structured Nonconvex and Nonsmooth Optimization: Algorithms and Iteration Complexity Analysis," 2016. [Online]. Available: http://arxiv.org/abs/1605.02408

[22] R. Tibshirani, "Regression shrinkage and selection via the lasso: a retrospective," Journal of the Royal Statistical Society: Series B (Statistical Methodology), vol. 73, no. 3, pp. 273-282, Jun. 2011.

[23] J. Fan and R. Li, "Variable Selection via Nonconcave Penalized Likelihood and its Oracle Properties," Journal of the American Statistical Association, vol. 96, no. 456, pp. 1348-1360, Dec. 2001.

[24] E. J. Candès, M. B. Wakin, and S. P. Boyd, "Enhancing Sparsity by Reweighted L1 Minimization," Journal of Fourier Analysis and Applications, vol. 14, no. 5-6, pp. 877-905, Dec. 2008.

[25] T. Zhang, "Analysis of Multi-stage Convex Relaxation for Sparse Regularization," Journal of Machine Learning Research, vol. 11, pp. 1081-
1107, 2010.

[26] J. Weston, A. Elisseeff, B. Scholkopf, and M. Tipping, "The use of zero-norm with linear models and kernel methods," Journal of Machine Learning Research, vol. 3, pp. 1439-1461, 2003.

[27] G. Gasso, A. Rakotomamonjy, and S. Canu, "Recovering sparse signals with a certain family of nonconvex penalties and DC programming," IEEE Transactions on Signal Processing, vol. 57, no. 12, pp. 46864698, Dec. 2009

[28] Y. Sun, P. Babu, and D. P. Palomar, "Majorization-Minimization Algorithms in Signal Processing, Communications, and Machine Learning," IEEE Transactions on Signal Processing, vol. 65, no. 3, pp. 794-816, Feb. 2017

[29] P. Gong, C. Zhang, Z. Lu, J. Huang, and J. Ye, "A General Iterative Shrinkage and Thresholding Algorithm for Non-convex Regularized Optimization Problems," in Proceedings of the 30th International Conference on Machine Learning, 2013, pp. 37-45.

[30] H. Attouch, J. Bolte, and B. F. Svaiter, "Convergence of descent methods for semi-algebraic and tame problems: Proximal algorithms, forwardbackward splitting, and regularized Gauss-Seidel methods," Mathematical Programming, vol. 137, no. 1-2, pp. 91-129, 2013.

[31] Y. Yang and M. Pesavento, "A parallel best-response algorithm with exact line search for nonconvex sparsity-regularized rank minimization,' in IEEE International Conference on Acoustics Speech and Signal Processing, apr 2018.

[32] Y. Yang, M. Pesavento, S. Chatzinotas, and B. Ottersten, "Successive Convex Approximation Algorithms for Sparse Signal Estimation with Nonconvex Regularizations," in IEEE Sensor Array and Multichannel Signal Processing Workshop (SAM), jul 2018.

[33] Q. Yao and J. T. Kwok, "Efficient Learning with a Family of Nonconvex Regularizers by Redistributing Nonconvexity," Journal of Machine Learning Research, vol. 18, pp. 1-52, 2018.

[34] S. Burer and R. D. Monteiro, "A nonlinear programming algorithm for solving semidefinite programs via low-rank factorization," Mathematical Programming, vol. 95, no. 2, pp. 329-357, Feb. 2003.

[35] B. Recht, M. Fazel, and P. A. Parrilo, "Guaranteed Minimum-Rank Solutions of Linear Matrix Equations via Nuclear Norm Minimization," SIAM Review, vol. 52, no. 3, pp. 471-501, Jan. 2010.

[36] E. Elhamifar and R. Vidal, "Sparse Subspace Clustering: Algorithm, Theory, and Applications," IEEE Transactions on Pattern Analysis and Machine Intelligence, vol. 35, no. 11, pp. 2765-2781, Nov. 2013.

[37] S. M. Robinson and R. H. Day, "A sufficient condition for continuity of optimal sets in mathematical programming," Journal of Mathematical Analysis and Applications, vol. 45, no. 2, pp. 506-511, Feb. 1974.

[38] Z. Yang, Z. Wang, H. Liu, Y. C. Eldar, and T. Zhang, "Sparse Nonlinear Regression: Parameter Estimation and Asymptotic Inference," 2016, in Proc. International Conference on Machine Learning (ICML). [Online]. Available: http://proceedings.mlr.press/v48/yangc16.pdf

[39] S. Wright, R. Nowak, and M. Figueiredo, "Sparse Reconstruction by Separable Approximation," IEEE Transactions on Signal Processing, vol. 57, no. 7, pp. 2479-2493, Jul. 2009.

[40] J. M. Ortega and W. C. Rheinboldt, Iterative solution of nonlinear equations in several variables. Academic, New York, 1970.

[41] C. Berge, Topological Spaces: Including a Treatment of Multi-Valued Functions, Vector Spaces and Convexity. Dover Publications, 1997. 